\title{
Regioselective $\beta$-Metalation of meso-Phosphanylporphyrins. Structure and Optical Properties of Porphyrin Dimers Linked by Peripherally Fused Phosphametallacycles
}

Yoshihiro Matano, ${ }^{*}{ }^{\dagger}$ Kazuaki Matsumoto ${ }^{\dagger}$ Yoshihide Nakao ${ }^{\dagger}$ Hidemitsu Uno, ${ }^{\dagger}$ Shigeyoshi Sakaki, ${ }^{\dagger}$ and Hiroshi Imahori ${ }^{\dagger} \S$

Department of Molecular Engineering, Graduate School of Engineering, Kyoto University, Nishikyo-ku, Kyoto 615-8510, Japan, Integrated Center for Sciences, Ehime University, Matsuyama 790-8577, Japan, and Institute for Integrated Cell-Material Sciences, Kyoto University, Nishikyo-ku, Kyoto 615-8510, Japan

Corresponding author: matano@scl.kyoto-u.ac.jp

${ }^{\dagger}$ Graduate School of Engineering, Kyoto University; ${ }^{*}$ Ehime University; ${ }^{\circledR}$ Institute for Integrated Cell-Material Sciences, Kyoto University

Contents:

General Remarks

SI-2

Synthetic Procedures and Characterization Data

SI-2

DFT Calculations

SI-6

Tables S1-S5

SI-8

Scheme S1:

SI-13

Figures S1-S27

SI-13 
General Remarks. ${ }^{1} \mathrm{H}$ and ${ }^{31} \mathrm{P}\left\{{ }^{1} \mathrm{H}\right\}$ NMR spectra were recorded using $\mathrm{CDCl}_{3}$ or $\mathrm{CD}_{2} \mathrm{Cl}_{2}$. The ${ }^{1} \mathrm{H}$ NMR spectra of new compounds are shown in Figures S20-S27. Chemical shifts are reported as the relative value vs. tetramethylsilane $\left({ }^{1} \mathrm{H}\right)$ and phosphoric acid $\left({ }^{31} \mathrm{P}\right)$, respectively. Matrix-assisted laser desorption/ionization (MALDI) time-of-flight mass spectra (TOF) were measured using CHCA as a matrix. Electrochemical measurements were performed using a glassy carbon working electrode, a platinum wire counter electrode, and an $\mathrm{Ag} / \mathrm{Ag}^{+}\left[0.01 \mathrm{M} \mathrm{AgNO}_{3}, 0.1 \mathrm{M} n \mathrm{Bu}_{4} \mathrm{NPF}_{6}(\mathrm{MeCN})\right]$ reference electrode. The potentials were calibrated with ferrocene/ferrocenium $\left[E_{\text {mid }}=+0.20 \mathrm{~V} \mathrm{vs} \mathrm{Ag/Ag}{ }^{+}\right.$. 5-Iodo-10,15,20-tris(3,5-di-t-butylphenyl)porphyrinatozinc(II) (1a) ${ }^{1}$ and 5-iodo-10,15,20-tris(2,4,6-trimethylphenyl)porphyrinatozinc(II) (1b) ${ }^{2}$ were prepared according to the reported procedures. The solvents used for the reactions were distilled from sodium benzophenone ketyl (THF) or calcium hydride $\left(\mathrm{CH}_{2} \mathrm{Cl}_{2}\right.$, toluene) under inert atmosphere before use. Other chemicals and solvents were of reagent grade quality, purchased commercially and used without further purification. Thin-layer chromatography and flash column chromatography were performed with Alt. 5554 DC-Alufolien Kieselgel $60 \mathrm{~F}_{254}$ (Merck) and Silica-gel 60N (Kanto Chemicals), respectively. All the reactions were performed under an argon atmosphere unless otherwise stated.

\section{5-Diphenylthiophosphoryl-10,15,20-tris(3,5-di-t-butylphenyl)porphyrinatozinc(II) (4a): A 30-mL} of flask containing 1a $(222 \mathrm{mg}, 0.209 \mathrm{mmol})$ and $\mathrm{Pd}(\mathrm{OAc})_{2}(10.9 \mathrm{mg}, 0.0486 \mathrm{mmol})$ was evacuated in vacuo and then filled with argon. The same manipulation was repeated three times. THF (11 mL), $\operatorname{MeCN}(8.8 \mathrm{~mL})$, triethylamine $(123 \mu \mathrm{L}, 0.89 \mathrm{mmol})$, and diphenylphosphine $(75 \mu \mathrm{L}, 0.43 \mathrm{mmol})$ were added via syringes to the flask, and the resulting mixture was stirred at $80{ }^{\circ} \mathrm{C}$ (bath temperature) for 18 h. After checking the consumption of $1 \mathbf{a}$ by TLC, $\mathrm{S}_{8}(13 \mathrm{mg}, 0.050 \mathrm{mmol})$ was added to the flask, and the mixture was stirred for an additional 3 h. The resulting mixture was filtered through a Celite bed, and the filtrate was concentrated under reduced pressure to leave a solid, which was then chromatographed on silica gel using hexane and $\mathrm{CH}_{2} \mathrm{Cl}_{2}$ as eluents. The bluish purple fraction $\left(R_{\mathrm{f}}=\right.$ 0.36 ; hexane/AcOEt $=5 / 1)$ was collected, concentrated, and reprecipitated from $\mathrm{CH}_{2} \mathrm{Cl}_{2} / \mathrm{MeOH}$ to give

1 Odobel, F.; Suzenet, F.; Blart, E.; Quintard, J.-P. Org. Lett. 2000, 2, 131-133.

2 Shultz, D. A.; Gwaltney, K. P.; Lee, H. J. Org. Chem. 1998, 63, 769-774. 
4a as a purple solid (221 mg, 92\%). ${ }^{1} \mathrm{H}$ NMR (400 MHz, $\left.\mathrm{CDCl}_{3}\right) \delta 1.48\left(\mathrm{~s}, 36 \mathrm{H} ; \mathrm{CH}_{3}\right), 1.51(\mathrm{~s}, 18 \mathrm{H}$; $\left.\mathrm{CH}_{3}\right), 7.20-7.35(4 \mathrm{H} ; m-\mathrm{Ph}), 7.38(\mathrm{t}, 2 \mathrm{H}, J=6.8 \mathrm{~Hz} ; p-\mathrm{Ph}), 7.73(\mathrm{~s}, 2 \mathrm{H} ; p-\mathrm{Ar}-\mathrm{H}), 7.78(\mathrm{~s}, 1 \mathrm{H} ; p-\mathrm{Ar}-\mathrm{H})$, $7.89(\mathrm{~m}, 4 \mathrm{H} ; o-\mathrm{Ph}), 7.93(\mathrm{~d}, 4 \mathrm{H}, J=1.2 \mathrm{~Hz} ; o-\mathrm{Ar}-\mathrm{H}), 8.01(\mathrm{~d}, 2 \mathrm{H}, J=1.2 \mathrm{~Hz} ; o-\mathrm{Ar}-\mathrm{H}), 8.52(\mathrm{~d}, 2 \mathrm{H}, J=$ $4.8 \mathrm{~Hz} ; \beta-\mathrm{H}), 8.85(\mathrm{~d}, 2 \mathrm{H}, J=5.2 \mathrm{~Hz} ; \beta-\mathrm{H}), 8.90(\mathrm{~d}, 2 \mathrm{H}, J=5.2 \mathrm{~Hz} ; \beta-\mathrm{H}), 9.12(\mathrm{~d}, 2 \mathrm{H}, J=4.8 \mathrm{~Hz}$; $\beta$-H); ${ }^{31} \mathrm{P}$ NMR (162 MHz, $\mathrm{CDCl}_{3}$ ) $\delta 40.1$; MS (MALDI-TOF) $m / z, 1154.5\left([\mathrm{M}+\mathrm{H}]^{+}, 100 \%\right)$; UV/Vis (toluene) $\lambda_{\max }(\varepsilon) 434$ (280000), 560 (13100), $600 \mathrm{~nm}\left(8700 \mathrm{M}^{-1} \mathrm{~cm}^{-1}\right)$.

\section{5-Diphenylthiophosphoryl-10,15,20-tris(2,4,6-trimethylphenyl)porphyrinatozinc(II) (4b): This} compound was prepared from $\mathbf{1 b}(112 \mathrm{mg}, 0.132 \mathrm{mmol}), \mathrm{Pd}(\mathrm{OAc})_{2}(5.9 \mathrm{mg}, 0.026 \mathrm{mmol})$, triethylamine $(73 \mu \mathrm{L}, 0.53 \mathrm{mmol})$, diphenylphosphine (46 $\mu \mathrm{L}, 0.21 \mathrm{mmol})$, and $\mathrm{S}_{8}(10 \mathrm{mg}, 0.039 \mathrm{mmol})$ according to a similar procedure described for the synthesis of $\mathbf{4 a}$. The bluish purple fraction $\left(R_{\mathrm{f}}=\right.$ 0.55; hexane/AcOEt $=5 / 1)$ was collected, concentrated, and reprecipitated from $\mathrm{CH}_{2} \mathrm{Cl}_{2} / \mathrm{MeOH}$ to give 4b (108 mg, 87\%). ${ }^{1} \mathrm{H}$ NMR (400 MHz, $\left.\mathrm{CD}_{2} \mathrm{Cl}_{2}\right) \delta 1.77\left(\mathrm{~s}, 12 \mathrm{H} ; o-\mathrm{Ar}-\mathrm{CH}_{3}\right), 1.86\left(\mathrm{~s}, 6 \mathrm{H} ; o-\mathrm{Ar}-\mathrm{CH}_{3}\right)$, 2.56 (s, 6H; p-Ar-CH $\mathrm{CH}_{3}$, 2.60 (s, 3H; p-Ar- $\mathrm{CH}_{3}$ ), 7.22 (s, 4H; m-Ar-H), 7.23 (m, 4H; m-Ph), 7.27 (s, 2H; $m$-Ar-H), 7.36 (t, 2H, $J=7.2 \mathrm{~Hz} ; p-\mathrm{Ph}), 7.71(\mathrm{~m}, 4 \mathrm{H} ; o-\mathrm{Ph}), 8.22(\mathrm{~d}, 2 \mathrm{H}, J=4.8 \mathrm{~Hz} ; \beta-\mathrm{H}), 8.52(\mathrm{~d}, 2 \mathrm{H}$, $J=4.8 \mathrm{~Hz} ; \beta-\mathrm{H}), 8.61(\mathrm{~d}, 2 \mathrm{H}, J=4.8 \mathrm{~Hz} ; \beta-\mathrm{H}), 8.99(\mathrm{~d}, 2 \mathrm{H}, J=4.8 \mathrm{~Hz} ; \beta-\mathrm{H}) ;{ }^{31} \mathrm{P} \mathrm{NMR}(162 \mathrm{MHz}$, $\left.\mathrm{CDCl}_{3}\right) \delta 39.0 ; \mathrm{MS}(\mathrm{MALDI}-\mathrm{TOF}) \mathrm{m} / z=944\left([\mathrm{M}+\mathrm{H}]^{+}, 100 \%\right)$; UV/Vis (toluene) $\lambda_{\max }(\varepsilon) 433$ (363000), 559 (16200), $598 \mathrm{~nm}\left(10600 \mathrm{M}^{-1} \mathrm{~cm}^{-1}\right)$.

\section{5-Diphenylphosphanyl-10,15,20-tris(3,5-di-t-butylphenyl)porphyrinatozinc(II) (2a): A 50-mL of}

flask containing 4a (165 mg, $0.143 \mathrm{mmol})$ was evacuated in vacuo and then filled with argon. The same manipulation was repeated three times. Toluene $(20 \mathrm{~mL})$ and $\mathrm{P}\left(\mathrm{NMe}_{2}\right)_{3}(1.82 \mathrm{~mL}, 10.0 \mathrm{mmol})$ were added via syringes to the flask, and the resulting mixture was stirred at $130{ }^{\circ} \mathrm{C}$ (bath temperature). After 144 h, 4a was consumed completely (checked by TLC). The solvent was concentrated under reduced pressure to leave a solid residue, which was reprecipitated from $\mathrm{CH}_{2} \mathrm{Cl}_{2} / \mathrm{MeOH}$ under argon atmosphere to give $\mathbf{2 a}$ as a purple solid (150 mg, 93\%). ${ }^{1} \mathrm{H}$ NMR $\left(400 \mathrm{MHz}, \mathrm{CDCl}_{3}\right) \delta 1.44(\mathrm{~s}, 36 \mathrm{H}$; $\left.\mathrm{CH}_{3}\right), 1.45\left(\mathrm{~s}, 18 \mathrm{H} ; \mathrm{CH}_{3}\right), 7.16-7.19(6 \mathrm{H} ; m-\mathrm{Ph}, p-\mathrm{Ph}), 7.58(\mathrm{t}, 4 \mathrm{H}, J=6.8 \mathrm{~Hz} ; o-\mathrm{Ph}), 7.70(\mathrm{t}, 2 \mathrm{H}, J=$ $2.0 \mathrm{~Hz} ; p-\mathrm{Ar}-\mathrm{H}), 7.71(\mathrm{t}, 1 \mathrm{H}, J=1.5 \mathrm{~Hz} ; p-\mathrm{Ar}-\mathrm{H}), 7.97(\mathrm{~d}, 4 \mathrm{H}, J=2.0 \mathrm{~Hz} ; o-\mathrm{Ar}-\mathrm{H}), 7.99(\mathrm{~d}, 2 \mathrm{H}, J=$ $1.5 \mathrm{~Hz} ; o-\mathrm{Ar}-\mathrm{H}), 8.83(\mathrm{~d}, 2 \mathrm{H}, J=4.8 \mathrm{~Hz} ; \beta-\mathrm{H}), 8.85(\mathrm{~d}, 2 \mathrm{H}, J=5.2 \mathrm{~Hz} ; \beta-\mathrm{H}), 8.90(\mathrm{~d}, 2 \mathrm{H}, J=5.2 \mathrm{~Hz}$; 
$\beta-\mathrm{H}), 9.86(\mathrm{~d}, 2 \mathrm{H}, J=4.8 \mathrm{~Hz} ; \beta-\mathrm{H}) ;{ }^{31} \mathrm{P} \mathrm{NMR}\left(162 \mathrm{MHz}, \mathrm{CDCl}_{3}\right) \delta-5.4 ; \mathrm{UV} /$ Vis (toluene) $\lambda_{\max }(\varepsilon) 426$ (260000), 555 (13500), $596 \mathrm{~nm}\left(6400 \mathrm{M}^{-1} \mathrm{~cm}^{-1}\right)$.

\section{5-Diphenylphosphanyl-10,15,20-tris(2,4,6-trimethylphenyl)porphyrinatozinc(II) (2b): This}

compound was prepared in 95\% yield $(96 \mathrm{mg})$ from $\mathbf{3 b}(105 \mathrm{mg}, 0.111 \mathrm{mmol}), \mathrm{P}\left(\mathrm{NMe}_{2}\right)_{3}(1.0 \mathrm{~mL}, 5.6$ $\mathrm{mmol})$, and toluene $(50 \mathrm{~mL})$ according to a similar procedure $\left(130^{\circ} \mathrm{C}, 14 \mathrm{~h}\right)$ described for the synthesis of 2a. ${ }^{1} \mathrm{H}$ NMR (400 MHz, $\left.\mathrm{CDCl}_{3}\right) \delta 1.82\left(\mathrm{~s}, 18 \mathrm{H} ; o-\mathrm{Ar}-\mathrm{CH}_{3}\right), 2.60\left(\mathrm{~s}, 6 \mathrm{H} ; p-\mathrm{Ar}^{-\mathrm{CH}_{3}}\right), 2.61(\mathrm{~s}, 3 \mathrm{H}$; $p$-Ar- $\left.\mathrm{CH}_{3}\right), 7.21-7.26(12 \mathrm{H} ; m-\mathrm{Ar}-\mathrm{H}, m-\mathrm{Ph}, p-\mathrm{Ph}), 7.62$ (t, 4H, $\left.J=7.2 \mathrm{~Hz} ; o-\mathrm{Ph}\right), 8.55$ (d, 2H, $J=4.4$ $\mathrm{Hz} ; \beta-\mathrm{H}), 8.56(\mathrm{~d}, 2 \mathrm{H}, J=4.4 \mathrm{~Hz} ; \beta-\mathrm{H}), 8.60(\mathrm{~d}, 2 \mathrm{H}, J=4.4 \mathrm{~Hz} ; \beta-\mathrm{H}), 9.70(\mathrm{~d}, 2 \mathrm{H}, J=4.8 \mathrm{~Hz} ; \beta-\mathrm{H})$; ${ }^{31} \mathrm{P}$ NMR $\left(162 \mathrm{MHz}, \mathrm{CDCl}_{3}\right) \delta-6.0$; UV/Vis (toluene) $\lambda_{\max }(\varepsilon) 426$ (260000), 555 (13500), $596 \mathrm{~nm}$ $\left(6400 \mathrm{M}^{-1} \mathrm{~cm}^{-1}\right)$.

$\boldsymbol{\beta}$-Palladioporphyrins (5a and 6a): A mixture of 2a (51 mg, $0.045 \mathrm{mmol}), \mathrm{Pd}(\mathrm{OAc})_{2}(6.1 \mathrm{mg}, 0.023$ $\mathrm{mmol}$ ), and toluene $(4 \mathrm{~mL})$ was stirred at $50{ }^{\circ} \mathrm{C}$ (bath temperature) in the dark for $24 \mathrm{~h}$. The solvent was concentrated under reduced pressure to leave a solid, which was chromatographed on silica gel using hexane and $\mathrm{CH}_{2} \mathrm{Cl}_{2}$ as eluents. The purple fractions $\left(R_{\mathrm{f}}=0.45\right.$ and 0.70 in hexane $\left./ \mathrm{AcOEt}=5 / 1\right)$ were collected, concentrated, and recrystallized from $\mathrm{CH}_{2} \mathrm{Cl}_{2} / \mathrm{MeOH}$ to give $\mathbf{5 a}$ and $\mathbf{6 a}$ in $55 \%$ and $23 \%$ yield, respectively. When a toluene solution of one equiv of $\mathbf{2 a}$ was slowly added to a solution of $\mathrm{Pd}(\mathrm{OAc})_{2}$ in refluxing toluene, 6a was formed predominantly in $73 \%$ yield. 5a: ${ }^{1} \mathrm{H} \mathrm{NMR}(400 \mathrm{MHz}$, $\left.\mathrm{CD}_{2} \mathrm{Cl}_{2}\right) \delta 1.52(\mathrm{~s}, 36 \mathrm{H}), 1.54(\mathrm{~s}, 36 \mathrm{H}), 1,59(\mathrm{~s}, 36 \mathrm{H}), 7.22(\mathrm{t}, 8 \mathrm{H}, J=7.6 \mathrm{~Hz} ; m-\mathrm{Ph}), 7.31(\mathrm{t}, 4 \mathrm{H}, J=$ $7.6 \mathrm{~Hz} ; p$-Ph), 7.82 (s, 2H; p-Ar), 7.83 (s, 2H; p-Ar), 7.84 (s, 2H; p-Ar), 8.01 (s, 4H; o-Ar), 8.08 (s, 8H; $o$-Ar), $8.40(\mathrm{~m}, 8 \mathrm{H} ; o-\mathrm{Ph}), 8.70(\mathrm{~d}, 2 \mathrm{H}, J=4.8 \mathrm{~Hz} ; \beta-\mathrm{H}), 8.88-8.91(8 \mathrm{H} ; \beta-\mathrm{H}), 9.05(\mathrm{~d}, 2 \mathrm{H}, J=4.4 \mathrm{~Hz}$; $\beta$-H), $9.10(\mathrm{~s}, 2 \mathrm{H} ; \beta-\mathrm{H}) ;{ }^{31} \mathrm{P}$ NMR $\left(162 \mathrm{MHz}, \mathrm{CD}_{2} \mathrm{Cl}_{2}\right) \delta 51.1$; MS (MALDI-TOF): $m / z 2349.5\left([\mathrm{M}+\mathrm{H}]^{+}\right.$, 100\%); UV/Vis (toluene) $\lambda_{\max }(\varepsilon) 426$ (427000), 554 (58600), 583 (15300), $601 \mathrm{~nm}\left(31200 \mathrm{M}^{-1} \mathrm{~cm}^{-1}\right)$. 6a: ${ }^{1} \mathrm{H}$ NMR (400 MHz, $\left.\mathrm{CD}_{2} \mathrm{Cl}_{2}\right) \delta 1.50$ (s, 36H), 1.53 (s, 36H), 1.63 (s, 36 H), 7.41-7.47 (12H; $m$ - $\mathrm{Ph}$, p-Ph), 7.81 (s, 2H; p-Ar), 7.84 (s, 2H; p-Ar), 7.91 (s, 2H; p-Ar), 8.02 (d, 4H, J = 2.0 Hz; o-Ar), 8.08 (d, $4 \mathrm{H}, J=2.0 \mathrm{~Hz} ; o-\mathrm{Ar}), 8.19$ (d, $4 \mathrm{H}, J=2.0 \mathrm{~Hz} ; o-\mathrm{Ar}), 8.23(\mathrm{~m}, 8 \mathrm{H} ; o-\mathrm{Ph}), 8.78(\mathrm{~d}, 2 \mathrm{H}, J=4.4 \mathrm{~Hz} ; \beta-\mathrm{H})$, $8.83(\mathrm{~d}, 2 \mathrm{H}, J=4.8 \mathrm{~Hz} ; \beta-\mathrm{H}), 8.88(\mathrm{~d}, 2 \mathrm{H}, J=4.4 \mathrm{~Hz} ; \beta-\mathrm{H}), 8.92(\mathrm{~d}, 2 \mathrm{H}, J=4.4 \mathrm{~Hz} ; \beta-\mathrm{H}), 8.97(\mathrm{~d}, 2 \mathrm{H}$, $J=4.4 \mathrm{~Hz} ; \beta-\mathrm{H}), 9.01(\mathrm{~d}, 2 \mathrm{H}, J=4.4 \mathrm{~Hz} ; \beta-\mathrm{H}), 9.14(\mathrm{~d}, 2 \mathrm{H}, J=3.2 \mathrm{~Hz} ; \beta-\mathrm{H}) ;{ }^{31} \mathrm{P} \mathrm{NMR}(162 \mathrm{MHz}$, 
$\left.\mathrm{CDCl}_{3}\right) \delta 50.5 ; \mathrm{MS}(\mathrm{MALDI}-\mathrm{TOF}) \mathrm{m} / z 2574\left([\mathrm{M}+\mathrm{H}]^{+}, 100 \%\right) ; \mathrm{UV} / \mathrm{Vis}$ (toluene) $\lambda_{\max }(\varepsilon) 438(548000)$, 552 (37600), $582(14200), 601 \mathrm{~nm}\left(22400 \mathrm{M}^{-1} \mathrm{~cm}^{-1}\right)$.

$\boldsymbol{\beta}$-Palladioporphyrin (5b): A mixture of $\mathbf{2 b}(46.9 \mathrm{mg}, 0.0514 \mathrm{mmol}), \mathrm{Pd}(\mathrm{OAc})_{2}(8.9 \mathrm{mg}, 0.040 \mathrm{mmol})$, and toluene $(10 \mathrm{~mL})$ was stirred at room temperature in the dark for $1.5 \mathrm{~h}$. The solvent was concentrated under reduced pressure to leave a solid, which was then chromatographed on silica gel using hexane and $\mathrm{CH}_{2} \mathrm{Cl}_{2}$ as eluents. The reddish orange fraction $\left(R_{\mathrm{f}}=0.61\right.$; hexane/AcOEt $\left.=5 / 1\right)$ was collected, concentrated, and reprecipitated from $\mathrm{CH}_{2} \mathrm{Cl}_{2} / \mathrm{MeOH}$ to give $\mathbf{5 b}(34.7 \mathrm{mg}, 70 \%) .{ }^{1} \mathrm{H}$ NMR $\left(400 \mathrm{MHz}, \mathrm{CD}_{2} \mathrm{Cl}_{2}\right) \delta 1.82\left(\mathrm{~s}, 12 \mathrm{H} ; o-\mathrm{Ar}-\mathrm{CH}_{3}\right), 1.83\left(\mathrm{~s}, 12 \mathrm{H} ; o-\mathrm{Ar}-\mathrm{CH}_{3}\right), 1.85\left(\mathrm{~s}, 12 \mathrm{H} ; o-\mathrm{Ar}-\mathrm{CH}_{3}\right), 2.61$ (s, 12H; p-Ar- $\mathrm{CH}_{3}$ ), 2.82 (s, 6H; p-Ar- $\mathrm{CH}_{3}$ ), 7.23 (t, 8H, J = 7.2 Hz; m-Ph), 7.27 (s, 4H; m-Ar-H), 7.28 (s, 4H; m-Ar-H), 7.35 (s, 4H; m-Ar-H), 7.37 (t, 4H, J = $7.2 \mathrm{~Hz} ; p-\mathrm{Ph}), 8.15(\mathrm{~m}, 8 \mathrm{H} ; o-\mathrm{Ph}), 8.56$ (d, 2H, $J=4.4 \mathrm{~Hz} ; \beta-\mathrm{H}), 8.57(\mathrm{~d}, 2 \mathrm{H}, J=6.8 \mathrm{~Hz} ; \beta-\mathrm{H}), 8.62(\mathrm{~d}, 2 \mathrm{H}, J=4.8 \mathrm{~Hz} ; \beta-\mathrm{H}), 8.65(\mathrm{~d}, 2 \mathrm{H}, J=6.4 \mathrm{~Hz}$; $\beta-\mathrm{H}), 8.66(\mathrm{~d}, 2 \mathrm{H}, J=6.4 \mathrm{~Hz} ; \beta-\mathrm{H}), 8.67(\mathrm{~d}, 2 \mathrm{H}, J=4.4 \mathrm{~Hz} ; \beta-\mathrm{H}), 8.96(\mathrm{~d}, 2 \mathrm{H}, J=4.0 \mathrm{~Hz} ; \beta-\mathrm{H}) ;{ }^{31} \mathrm{P}$ NMR (162 MHz, $\left.\mathrm{CDCl}_{3}\right) \delta$ 48.6; MS (FAB) $m / z 1929.5\left([\mathrm{M}+\mathrm{H}]^{+}, 100 \%\right) ; \mathrm{UV} /$ Vis (toluene) $\lambda_{\max }(\varepsilon)$ 422 (235000), 440 (187000), 556 (34000), 583 (12100), $600 \mathrm{~nm}\left(18900 \mathrm{M}^{-1} \mathrm{~cm}^{-1}\right)$.

$\boldsymbol{\beta}$-Platinioporphyrin (7a): A mixture of $\mathbf{2 a}(32.4 \mathrm{mg}, 0.027 \mathrm{mmol}), \mathrm{PtCl}_{2}(\mathrm{cod})(5.0 \mathrm{mg}, 0.013 \mathrm{mmol})$, triethylamine (40 $\mu \mathrm{l}, 0.18 \mathrm{mmol})$, and $\mathrm{CH}_{2} \mathrm{Cl}_{2}(4 \mathrm{~mL})$ was stirred for $3 \mathrm{~h}$ in the dark. The solvent was concentrated under reduced pressure to leave a solid, which was chromatographed on silica gel using hexane and $\mathrm{CH}_{2} \mathrm{Cl}_{2}$ as eluents. The brown fraction $\left(R_{\mathrm{f}}=0.47\right.$ in hexane/AcOEt $\left.=9 / 1\right)$ was collected, concentrated, and recrystallized from $\mathrm{CH}_{2} \mathrm{Cl}_{2} / \mathrm{MeOH}$ to give 7a as a brownish purple solid in $62 \%$ yield. ${ }^{1} \mathrm{H}$ NMR (400 MHz, $\left.\mathrm{CDCl}_{3}\right) \delta 1.49$ (s, 36H), 1.52 (s, 36H), 1.61 (s, $\left.36 \mathrm{H}\right), 7.17$ (m, 8H; m-Ph), 7.26 (m, 4H; p-Ph), 7.76 (s, 2H; p-Ar), 7.77 (s, 2H; p-Ar), 7.94 (s, 2H; p-Ar), 7.99 (s, 4H;o-Ar), 8.06 (s, 4H; $o$-Ar), 8.08 (s, 4H; o-Ar), $8.44(\mathrm{~m}, 8 \mathrm{H} ; o-\mathrm{Ph}), 8.67(\mathrm{~d}, 2 \mathrm{H}, J=4.8 \mathrm{~Hz} ; \beta-\mathrm{H}), 8.90(\mathrm{~d}, 2 \mathrm{H}, J=4.4 \mathrm{~Hz}$; $\beta-\mathrm{H}), 8.92(\mathrm{~d}, 2 \mathrm{H}, J=4.4 \mathrm{~Hz} ; \beta-\mathrm{H}), 8.93(\mathrm{~d}, 2 \mathrm{H}, J=4.4 \mathrm{~Hz} ; \beta-\mathrm{H}), 8.99(\mathrm{~d}, 4 \mathrm{H}, J=5.2 \mathrm{~Hz} ; \beta-\mathrm{H}), 9.10$ $(\mathrm{s}, 2 \mathrm{H} ; \beta-\mathrm{H}) ;{ }^{31} \mathrm{P} \mathrm{NMR}\left(162 \mathrm{MHz}, \mathrm{CDCl}_{3}\right) \delta 46.7\left(\mathrm{~J}_{\mathrm{P}-\mathrm{Pt}}=2834 \mathrm{~Hz}\right) ; \mathrm{MS}(\mathrm{MALDI}-\mathrm{TOF}) \mathrm{m} / z 2438$ $\left([\mathrm{M}+\mathrm{H}]^{+}, 100 \%\right) ; \mathrm{UV} /$ Vis (toluene) $\lambda_{\max }(\varepsilon) 422$ (289000), 562 (38900), 592 (15700), $610 \mathrm{~nm}$ (27000 $\left.\mathrm{M}^{-1} \mathrm{~cm}^{-1}\right)$. 
Computational Details. Usual (21/11/41), (341/321/31), and (341/321/21) basis sets were used for $\mathrm{Zn}$ (up to $2 \mathrm{p}$ ), $\mathrm{Pd}$ (up to $3 \mathrm{~d}$ ), and $\mathrm{Pt}\left(\right.$ up to $4 \mathrm{f}$ ), respectively, with effective core potentials (ECP) ${ }^{3}$ employed to replace core electrons. For the other $\mathrm{H}, \mathrm{C}, \mathrm{N}$, and P, the 6-31G* basis sets were used. ${ }^{4}$ The geometry optimization was performed by the $\mathrm{B}^{2} \mathrm{LYP}^{5}$ method with above basis sets without any geometrical constraints, where X-ray structures in $C_{\mathrm{i}}$ symmetry were used as initial geometries. We calculated frequencies with the B3LYP method to ascertain that each optimized geometry was not in saddle but in equilibrium points. The optimized geometries of TPPZn, 8 , and 9 take $D_{2 d}, C_{i}$, and $C_{1}$ symmetries, respectively. The triplet instability of wavefunction was checked. The excited states and oscillator strengths are evaluated by the TD-B3LYP method, where forty excited states were solved. The effect of toluene solvent is estimated by the polarizable continuum model (PCM) method. ${ }^{6}$ All calculations were carried out with the Gaussian 03 package. ${ }^{7}$ Molecular orbitals with the isovalue of 0.05 are drawn by the Molekel 4.6. ${ }^{8}$ The absorption spectra in Figures S9-S14, S17, and S18 were drawn by gaussian fitting with the bandwidth of $0.15 \mathrm{eV}$. The PCM calculations indicate that Soret

3 (a) Wadt W. R.; Hay P. J. J. Chem. Phys. 1985, 82, 284-298. (b) Hay P. J.; Wadt W. R. J. Chem. Phys. 1985, 82, 299-310.

${ }^{4}$ (a) Hehre W. J.; Ditchfield R.; Pople J. A. J. Chem. Phys. 1972, 56, 2257-2261. (b) Dill J. D.; Pople J. A. J. Chem. Phys. 1975, 62, 2921-2923. (c) Hariharan P. C.; Pople J. A. Theoret. Chimica Acta 1973, $28,213-222$.

${ }^{5}$ (a) Becke, A. D. Phys Rev. A. 1988, 38, 3098-3100. (b) Becke, A. D. J. Chem. Phys. 1993, 98, 5648-5652. (c) Lee, C.; Yang, W.; Parr, R. G. Phys. Rev. B 1988, 37, 785-789.

${ }^{6}$ Cancès M. T.; Mennucci B.; Tomasi J. J. Chem. Phys. 1997, 107, 3032-3041.

${ }^{7}$ Gaussian 03, Revision C.02, Frisch, M. J.; Trucks, G. W.; Schlegel, H. B.; Scuseria, G. E.; Robb, M. A.; Cheeseman, J. R.; Montgomery, Jr., J. A.; Vreven, T.; Kudin, K. N.; Burant, J. C.; Millam, J. M.; Iyengar, S. S.; Tomasi, J.; Barone, V.; Mennucci, B.; Cossi, M.; Scalmani, G.; Rega, N.; Petersson, G. A.; Nakatsuji, H.; Hada, M.; Ehara, M.; Toyota, K.; Fukuda, R.; Hasegawa, J.; Ishida, M.; Nakajima, T.; Honda, Y.; Kitao, O.; Nakai, H.; Klene, M.; Li, X.; Knox, J. E.; Hratchian, H. P.; Cross, J. B.; Bakken, V.; Adamo, C.; Jaramillo, J.; Gomperts, R.; Stratmann, R. E.; Yazyev, O.; Austin, A. J.; Cammi, R.; Pomelli, C.; Ochterski, J. W.; Ayala, P. Y.; Morokuma, K.; Voth, G. A.; Salvador, P.; Dannenberg, J. J.; Zakrzewski, V. G.; Dapprich, S.; Daniels, A. D.; Strain, M. C.; Farkas, O.; Malick, D. K.; Rabuck, A. D.; Raghavachari, K.; Foresman, J. B.; Ortiz, J. V.; Cui, Q.; Baboul, A. G.; Clifford, S.; Cioslowski, J.; Stefanov, B. B.; Liu, G.; Liashenko, A.; Piskorz, P.; Komaromi, I.; Martin, R. L.; Fox, D. J.; Keith, T.; Al-Laham, M. A.; Peng, C. Y.; Nanayakkara, A.; Challacombe, M.; Gill, P. M. W.; Johnson, B.; Chen, W.; Wong, M. W.; Gonzalez, C.; and Pople, J. A.; Gaussian, Inc., Wallingford CT, 2004.

${ }^{8}$ MOLEKEL 4.0, P. Flükiger, H.P. Lüthi, S. Portmann, J. Weber, Swiss National Supercomputing Centre CSCS, Manno (Switzerland), 2000. 
band of TPPZn shifts to lower energy by $21 \mathrm{~nm}$ in toluene relative to that in vacuum but the small absorption around $550 \mathrm{~nm}$ little shifts to lower energy (only $3 \mathrm{~nm}$ ) in toluene (see Figures S9 and S10). Similarly, Soret and Q bands of $\mathbf{8}$ and $\mathbf{9}$ shift largely to lower energy in toluene (see, Figures S11-S14). Although calculated absorption bands with solvation effects shift to higher energy by $0.2-0.3 \mathrm{eV}$ from experimental ones, the theoretical spectra in toluene (Figures S10, S12, and S14) agree well with the experimental spectra (Figure 3) except for 7a, in which the calculated absorption spectra in Figure S14 do not exhibit the shoulder bands experimentally observed in the 450-500 nm region (Figure 3).

To know whether or not the $\mathrm{p}_{\pi}-\mathrm{d}_{\pi}$ orbital interaction between porphyrin and bridging Pd atom induces the splitting of Soret band of $\mathbf{8}, \beta$ - $\eta^{1}$-zinc-porphyrin (Zn-ref; see Table S5) was calculated as a reference. The geometry of $\mathbf{Z n}$-ref used for the TD-B3LYP calculation is identical to that of $\mathbf{8}$ optimized by the B3LYP method described above, in other words, the Pd atom in $\mathbf{8}$ is just replaced by the $\mathrm{Zn}$ atom in Zn-ref. The frontier orbitals, orbital energies, excitation spectra, and excitation energies of Zn-ref are summarized in Figures S15-S18 and Table S5. As shown in Figure S18 and Table S5, the splitting of Soret band of Zn-ref $(0.10 \mathrm{eV})$ is much smaller than that of the Pd-linked dimer 8 (ca. $0.15 \mathrm{eV}$ ). This result supports that the splitting of Soret-like band of the Pd-linked dimer 8 (5a) is indication of the $\mathrm{p}_{\pi}-\mathrm{d}_{\pi}$ orbital interaction. 
Table S1. Selected bond lengths (in $\AA$ ) and bond angles (in deg) of TPPZn, 8, and 9.

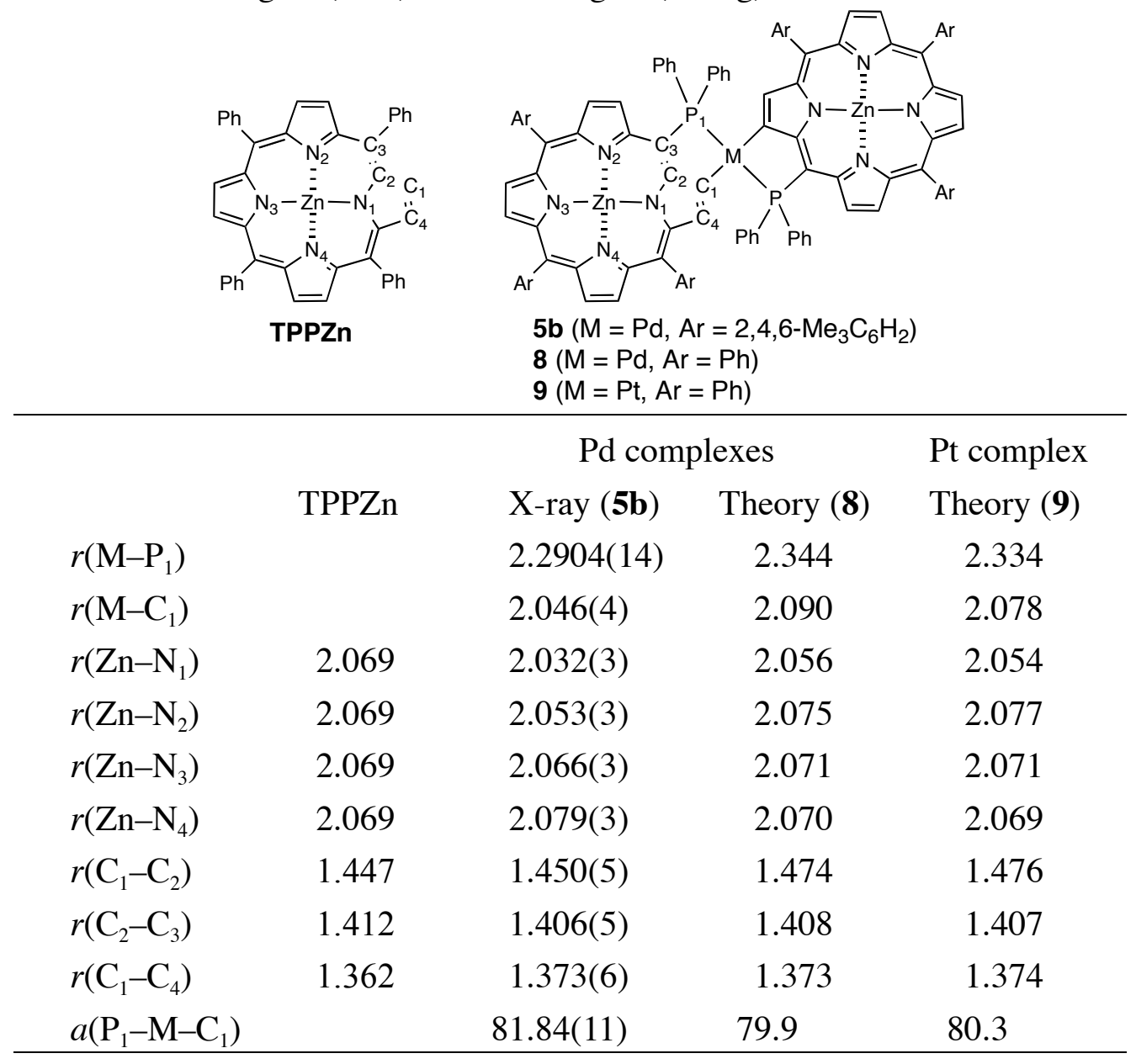


Table S2. Excitation energies and oscillator strengths of TPPZn calculated by the TD-B3LYP method. ${ }^{a}$

\begin{tabular}{|c|c|c|c|c|c|c|}
\hline State & $\begin{array}{l}\text { Excita } \\
(\mathrm{eV})\end{array}$ & $\begin{array}{l}\text { nergy } \\
(\mathrm{nm})\end{array}$ & $\begin{array}{l}\text { Oscillator } \\
\text { strength }\end{array}$ & & citation & Weight (\%) \\
\hline Withol & ation ef & & & & & \\
\hline 1 & 2.27 & 546 & 0.022 & HOMO & -> LUMO & 30 \\
\hline & & & & HOMO-1 & -> LUMO+1 & 21 \\
\hline 2 & 2.27 & 546 & 0.022 & HOMO & -> LUMO+1 & 30 \\
\hline & & & & HOMO-1 & $\rightarrow$ LUMO & 21 \\
\hline 3 & 3.25 & 382 & 1.340 & HOMO-1 & $\rightarrow$ LUMO & 20 \\
\hline & & & & HOMO & $\rightarrow$ LUMO+1 & 11 \\
\hline 4 & 3.25 & 382 & 1.340 & HOMO-1 & $\rightarrow$ LUMO+1 & 20 \\
\hline & & & & HOMO & -> LUMO & 11 \\
\hline With s & on effec & $\mathrm{M} \mathrm{me}$ & & & & \\
\hline 1 & 2.26 & 549 & 0.042 & HOMO & -> LUMO & 31 \\
\hline & & & & HOMO-1 & $\rightarrow$ LUMO+1 & 20 \\
\hline 2 & 2.26 & 549 & 0.042 & HOMO & $\rightarrow$ LUMO+1 & 31 \\
\hline & & & & HOMO-1 & $\rightarrow$ LUMO & 20 \\
\hline 3 & 3.08 & 403 & 1.700 & HOMO-1 & $\rightarrow$ LUMO & 22 \\
\hline & & & & HOMO & $\rightarrow$ LUMO+1 & 11 \\
\hline 4 & 3.08 & 403 & 1.700 & HOMO-1 & $\rightarrow$ LUMO+1 & 22 \\
\hline & & & & HOMO & -> LUMO & 11 \\
\hline
\end{tabular}

${ }^{a}$ The states whose oscillator strengths are less than 0.2 are not included except for Q bands. 
Table S3. Excitation energies and oscillator strengths of $\mathbf{8}$ calculated by the TD-B3LYP method. ${ }^{a}$

\begin{tabular}{|c|c|c|c|c|c|c|}
\hline \multirow[t]{2}{*}{ State } & \multicolumn{2}{|c|}{ Excitation energy } & \multirow{2}{*}{$\begin{array}{l}\text { Oscillator } \\
\text { strength }\end{array}$} & \multirow{2}{*}{\multicolumn{2}{|c|}{ Excitation }} & \multirow[t]{2}{*}{ Weight (\%) } \\
\hline & $(\mathrm{eV})$ & $(\mathrm{nm})$ & & & & \\
\hline \multicolumn{7}{|c|}{ Without solvation effects } \\
\hline \multirow[t]{2}{*}{1} & 2.24 & 553 & 0.085 & HOMO & $\rightarrow$ LUMO & 20 \\
\hline & & & & HOMO-2 & $\rightarrow$ LUMO+2 & 11 \\
\hline \multirow[t]{2}{*}{7} & 2.59 & 478 & 0.035 & HOMO-2 & $\rightarrow$ LUMO & 22 \\
\hline & & & & HOMO-3 & $\rightarrow$ LUMO+1 & 22 \\
\hline \multirow[t]{2}{*}{9} & 2.65 & 468 & 0.202 & HOMO & -> LUMO+2 & 23 \\
\hline & & & & HOMO-1 & -> LUMO+3 & 20 \\
\hline \multirow[t]{2}{*}{11} & 2.75 & 451 & 0.209 & HOMO-2 & $\rightarrow$ LUMO+2 & 28 \\
\hline & & & & HOMO-3 & $\rightarrow$ LUMO +3 & 12 \\
\hline 13 & 2.90 & 428 & 0.324 & HOMO-4 & $\rightarrow$ LUMO & 28 \\
\hline \multirow[t]{2}{*}{15} & 3.02 & 410 & 1.090 & HOMO-4 & $\rightarrow$ LUMO+2 & 15 \\
\hline & & & & HOMO-4 & -> LUMO & 10 \\
\hline \multirow[t]{2}{*}{17} & 3.14 & 394 & 1.110 & HOMO-4 & -> LUMO+2 & 19 \\
\hline & & & & HOMO-5 & -> LUMO+1 & 13 \\
\hline 20 & 3.20 & 388 & 0.521 & HOMO-5 & -> LUMO+1 & 28 \\
\hline 21 & 3.24 & 383 & 1.620 & HOMO-1 & $\rightarrow$ LUMO+3 & 6 \\
\hline 23 & 3.26 & 380 & 0.435 & HOMO-6 & $\rightarrow$ LUMO & 34 \\
\hline \multicolumn{7}{|c|}{ With solvation effects (PCM method) } \\
\hline \multirow[t]{3}{*}{1} & 2.231 & 556 & 0.152 & HOMO & $\rightarrow$ LUMO & 21 \\
\hline & & & & HOMO-1 & $\rightarrow$ LUMO+1 & 11 \\
\hline & & & & HOMO-2 & $\rightarrow$ LUMO+2 & 10 \\
\hline \multirow[t]{2}{*}{6} & 2.48 & 499 & 0.011 & HOMO-1 & $\rightarrow$ LUMO+1 & 22 \\
\hline & & & & HOMO & $\rightarrow$ LUMO & 20 \\
\hline \multirow[t]{2}{*}{7} & 2.59 & 478 & 0.116 & HOMO-3 & $\rightarrow$ LUMO+1 & 21 \\
\hline & & & & HOMO-2 & $\rightarrow$ LUMO & 21 \\
\hline \multirow[t]{2}{*}{9} & 2.64 & 470 & 0.362 & HOMO & $\rightarrow$ LUMO+2 & 24 \\
\hline & & & & HOMO-1 & $\rightarrow$ LUMO +3 & 18 \\
\hline 11 & 2.74 & 453 & 0.532 & HOMO-2 & $\rightarrow$ LUMO +2 & 30 \\
\hline \multirow[t]{2}{*}{13} & 2.88 & 430 & 0.658 & HOMO-4 & $\rightarrow$ LUMO & 20 \\
\hline & & & & HOMO-3 & $\rightarrow$ LUMO+3 & 18 \\
\hline \multirow[t]{2}{*}{15} & 2.96 & 418 & 2.135 & HOMO-3 & $\rightarrow$ LUMO+3 & 11 \\
\hline & & & & HOMO-4 & $\rightarrow$ LUMO & 10 \\
\hline 18 & 3.10 & 400 & 1.464 & HOMO-4 & $\rightarrow$ LUMO+2 & 19 \\
\hline 19 & 3.12 & 398 & 1.492 & HOMO-4 & $\rightarrow$ LUMO +2 & 16 \\
\hline
\end{tabular}

${ }^{a}$ The states whose oscillator strengths are less than 0.2 are not included except for $\mathrm{Q}$ bands. 
Table S4. Excitation energies and oscillator strengths of 9 calculated by the TD-B3LYP method. ${ }^{a}$

\begin{tabular}{|c|c|c|c|c|c|}
\hline \multirow[t]{2}{*}{ State } & \multicolumn{2}{|c|}{ Excitation energy } & \multirow{2}{*}{$\begin{array}{l}\text { Oscillator } \\
\text { strength }\end{array}$} & \multirow[t]{2}{*}{ Excitation } & \multirow[t]{2}{*}{ Weight $(\%)$} \\
\hline & $(\mathrm{eV})$ & $(\mathrm{nm})$ & & & \\
\hline \multicolumn{6}{|c|}{ Without solvation effects } \\
\hline 1 & 2.19 & 566 & 0.130 & HOMO $\rightarrow$ LUMO & 34 \\
\hline \multirow[t]{2}{*}{6} & 2.45 & 506 & 0.038 & HOMO-1 -> LUMO+1 & 13 \\
\hline & & & & HOMO-3 -> LUMO+1 & 13 \\
\hline \multirow[t]{2}{*}{7} & 2.56 & 485 & 0.494 & $\mathrm{HOMO} \rightarrow \mathrm{LUMO}+2$ & 15 \\
\hline & & & & HOMO-1 -> LUMO+1 & 13 \\
\hline \multirow[t]{2}{*}{15} & 2.94 & 422 & 0.214 & HOMO-4 $\rightarrow$ LUMO+2 & 28 \\
\hline & & & & HOMO-3 $\rightarrow$ LUMO+3 & 12 \\
\hline 17 & 3.15 & 394 & 2.400 & HOMO-4 -> LUMO+2 & 10 \\
\hline 21 & 3.26 & 380 & 1.750 & HOMO-1 $\rightarrow$ LUMO+3 & 7 \\
\hline \multicolumn{6}{|c|}{ With solvation effects (PCM method) } \\
\hline 1 & 2.18 & 568 & 0.216 & HOMO $\rightarrow$ LUMO & 35 \\
\hline \multirow[t]{2}{*}{6} & 2.45 & 506 & 0.083 & HOMO-3 -> LUMO+1 & 12 \\
\hline & & & & HOMO-1 $\rightarrow$ LUMO+1 & 11 \\
\hline \multirow[t]{2}{*}{7} & 2.54 & 489 & 0.807 & $\mathrm{HOMO} \rightarrow \mathrm{LUMO}+2$ & 16 \\
\hline & & & & HOMO-1 -> LUMO+1 & 15 \\
\hline \multirow[t]{2}{*}{8} & 2.58 & 481 & 0.061 & HOMO-2 -> LUMO & 28 \\
\hline & & & & HOMO-3 -> LUMO+1 & 10 \\
\hline \multirow[t]{2}{*}{11} & 2.73 & 454 & 0.178 & HOMO-2 $\rightarrow$ LUMO+2 & 24 \\
\hline & & & & HOMO-1 $\rightarrow$ LUMO+3 & 15 \\
\hline \multirow[t]{2}{*}{15} & 2.93 & 423 & 0.631 & $\mathrm{HOMO}-4 \rightarrow \mathrm{LUMO}+2$ & 18 \\
\hline & & & & HOMO-3 $\rightarrow$ LUMO+3 & 18 \\
\hline 17 & 3.06 & 405 & 2.535 & HOMO-4 -> LUMO+2 & 20 \\
\hline 19 & 3.12 & 397 & 2.488 & HOMO-4 -> LUMO & 8 \\
\hline
\end{tabular}

${ }^{a}$ The states whose oscillator strengths are less than 0.2 are not included except for $\mathrm{Q}$ bands.. 
Table S5. Excitation energies and oscillator strengths of Zn-ref calculated by the TD-B3LYP method. ${ }^{a}$

\begin{tabular}{|c|c|c|c|c|c|c|}
\hline \multirow[t]{2}{*}{ State } & \multicolumn{2}{|c|}{ Excitation energy } & \multirow{2}{*}{$\begin{array}{l}\text { Oscillator } \\
\text { strength }\end{array}$} & \multirow{2}{*}{\multicolumn{2}{|c|}{ Excitation }} & \multirow[t]{2}{*}{ Weight $(\%)$} \\
\hline & $(\mathrm{eV})$ & $(\mathrm{nm})$ & & & & \\
\hline \multicolumn{7}{|c|}{ Without solvation effects } \\
\hline 1 & 1.84 & 674 & 0.048 & HOMO & $\rightarrow$ LUMO+1 & 43 \\
\hline \multirow[t]{3}{*}{5} & 2.27 & 546 & 0.050 & HOMO-1 & $\rightarrow$ LUMO & 17 \\
\hline & & & & HOMO-2 & $\rightarrow$ LUMO+1 & 13 \\
\hline & & & & HOMO-3 & $\rightarrow$ LUMO+2 & 11 \\
\hline \multirow[t]{2}{*}{15} & 2.77 & 447 & 0.051 & HOMO-3 & $\rightarrow$ LUMO+2 & 26 \\
\hline & & & & HOMO-4 & -> LUMO+3 & 22 \\
\hline 17 & 3.04 & 408 & 0.133 & HOMO & $\rightarrow$ LUMO+4 & 41 \\
\hline 18 & 3.10 & 399 & 2.990 & HOMO-4 & $\rightarrow$ LUMO+3 & 13 \\
\hline 20 & 3.23 & 385 & 1.870 & HOMO-1 & $\rightarrow$ LUMO+3 & 7 \\
\hline \multirow[t]{2}{*}{23} & 3.30 & 376 & 0.354 & HOMO-6 & $\rightarrow$ LUMO+1 & 18 \\
\hline & & & & HOMO-5 & $\rightarrow$ LUMO & 12 \\
\hline \multicolumn{7}{|c|}{ With solvation effects (PCM method) } \\
\hline 1 & 1.81 & 685 & 0.068 & HOMO & $\rightarrow$ LUMO+1 & 43 \\
\hline \multirow[t]{3}{*}{5} & 2.26 & 548 & 0.092 & HOMO-1 & -> LUMO & 18 \\
\hline & & & & HOMO-2 & -> LUMO+1 & 14 \\
\hline & & & & HOMO-3 & $\rightarrow$ LUMO+2 & 10 \\
\hline \multirow[t]{2}{*}{11} & 2.64 & 470 & 0.013 & HOMO-4 & -> LUMO & 25 \\
\hline & & & & HOMO-3 & $\rightarrow$ LUMO+1 & 24 \\
\hline \multirow[t]{2}{*}{13} & 2.67 & 465 & 0.013 & HOMO-2 & $\rightarrow$ LUMO+2 & 25 \\
\hline & & & & HOMO-1 & $\rightarrow$ LUMO+3 & 24 \\
\hline \multirow[t]{2}{*}{15} & 2.78 & 446 & 0.151 & HOMO-3 & $\rightarrow$ LUMO+2 & 29 \\
\hline & & & & HOMO-4 & -> LUMO+3 & 19 \\
\hline 17 & 2.98 & 416 & 3.550 & HOMO-4 & $\rightarrow$ LUMO+3 & 18 \\
\hline 20 & 3.08 & 402 & 2.870 & HOMO-1 & $\rightarrow$ LUMO+3 & 9 \\
\hline
\end{tabular}

${ }^{a}$ The states whose oscillator strengths are less than 0.2 are not included except for $\mathrm{Q}$ bands.

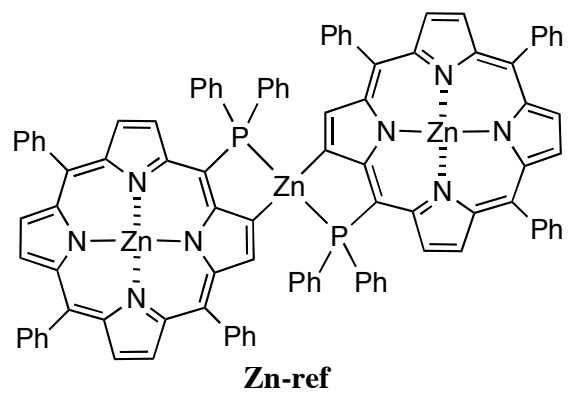




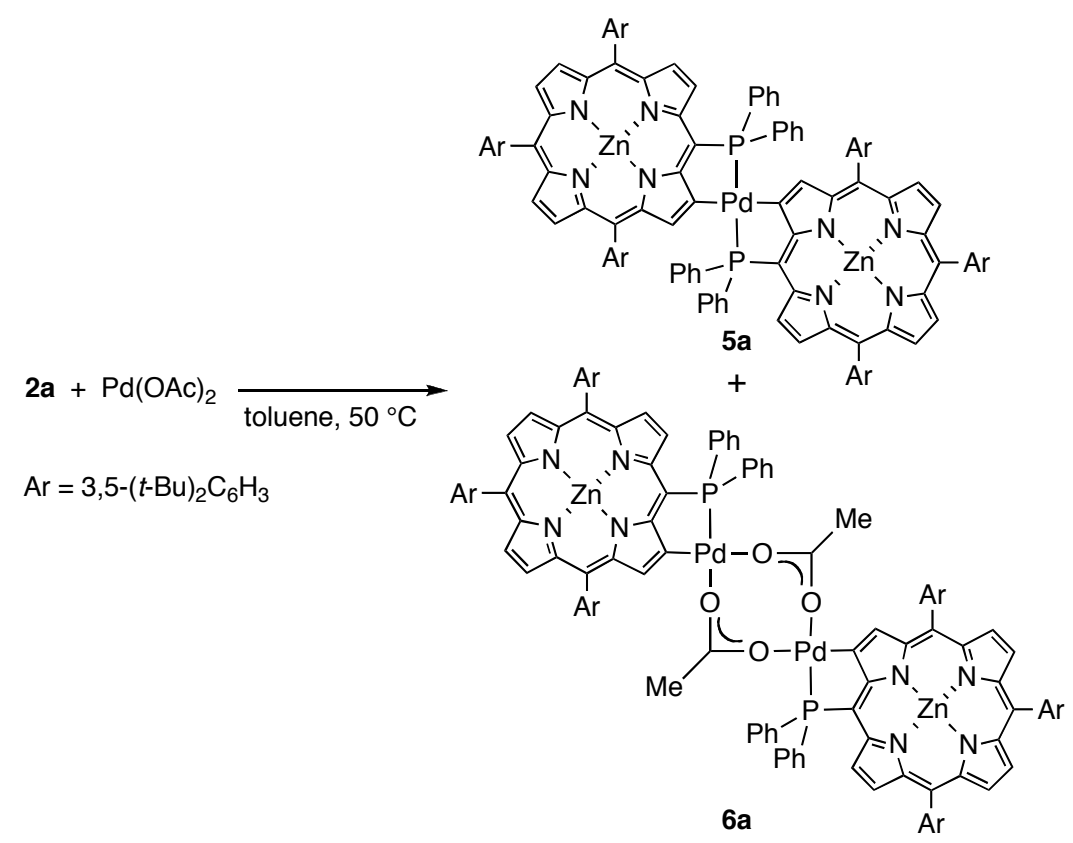

Scheme S1. Synthesis of palladium complexes $\mathbf{5 a}$ and $\mathbf{6 a}$.
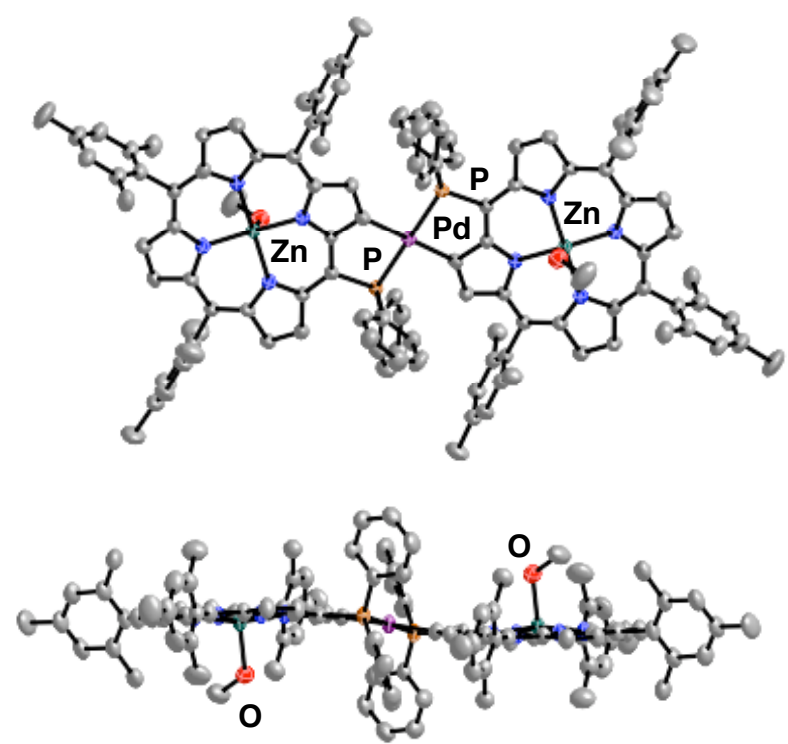

Figure S1. Top view (upper) and side view (lower) of $\mathbf{5 b}$ (30\% probability ellipsoids). Hydrogen atoms and solvents are omitted for clarity. 


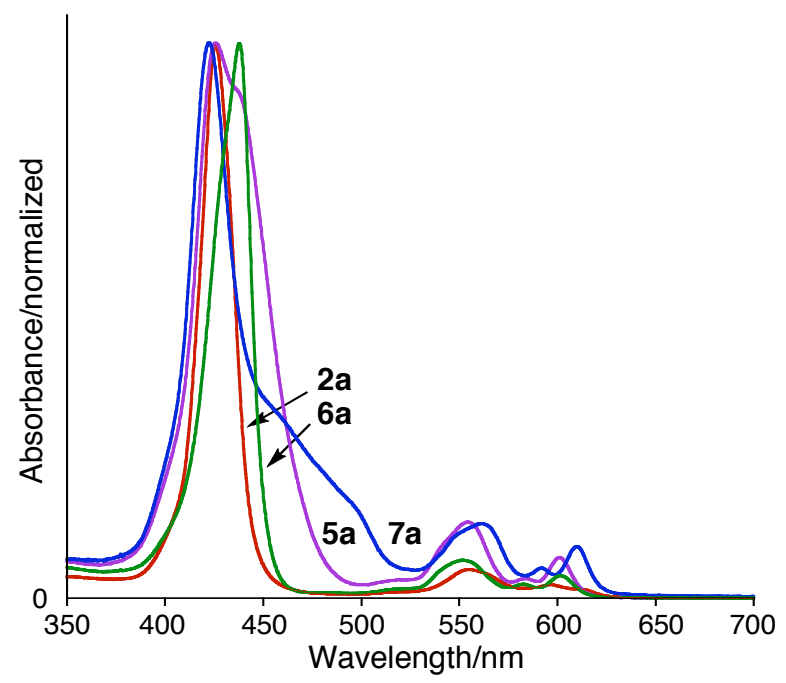

Figure S2. UV-vis absorption spectra of $\mathbf{2 a}$ (red), 5a (purple), 6a (green), and 7a (blue) in toluene. 


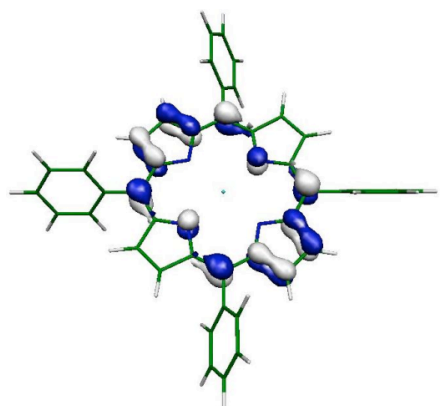

LUMO+1 (-2.14)

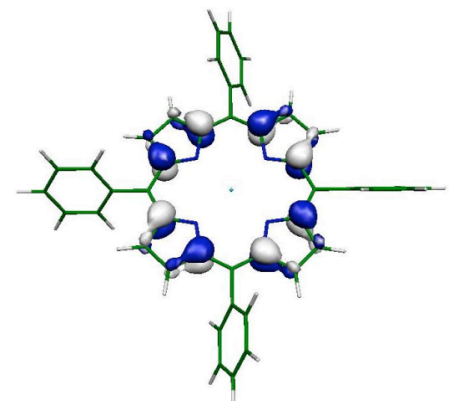

HOMO-1 (-5.15)

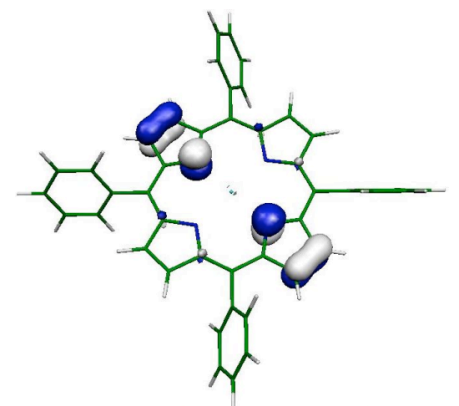

HOMO-4 (-6.45)

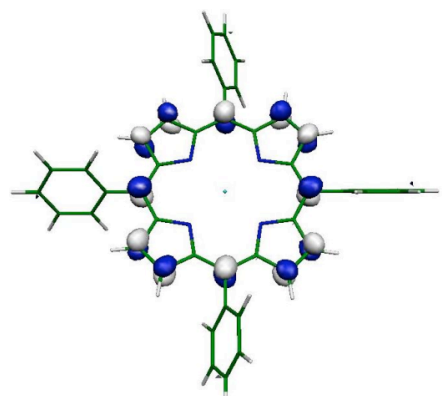

LUMO+2 (-0.62)

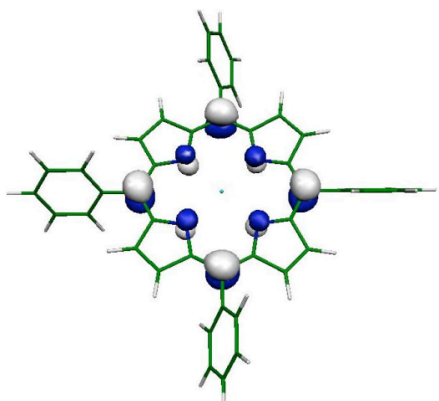

HOMO (-4.92)

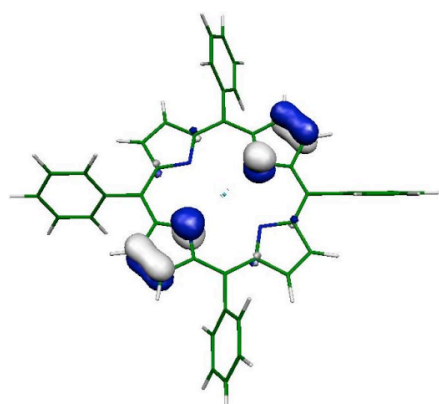

HOMO-3 (-6.45)

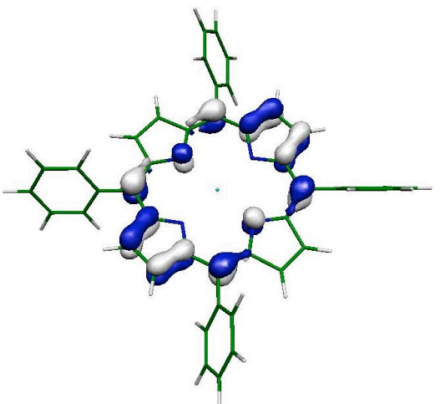

LUMO (-2.14)

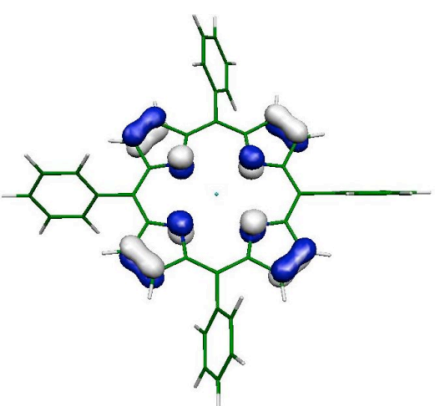

HOMO-2 (-6.41)

Figure S3. Molecular orbitals of TPPZn and their energies (in eV) calculated by the B3LYP method in gas phase. 


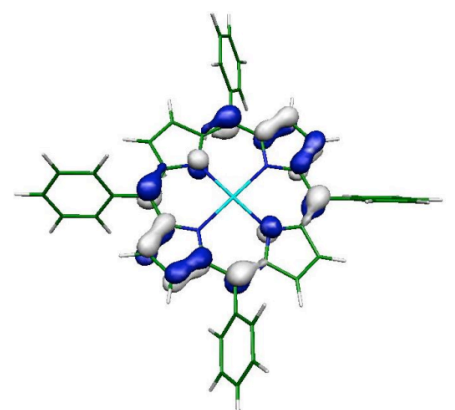

LUMO+1 (-2.19)

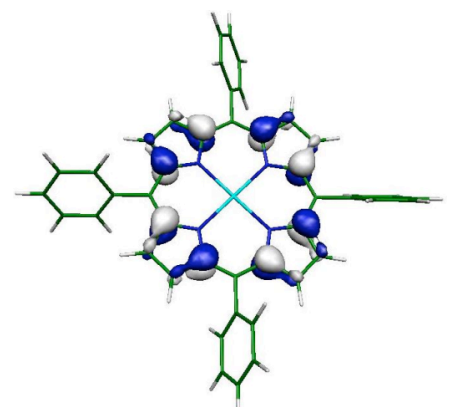

HOMO-1 (-5.20)

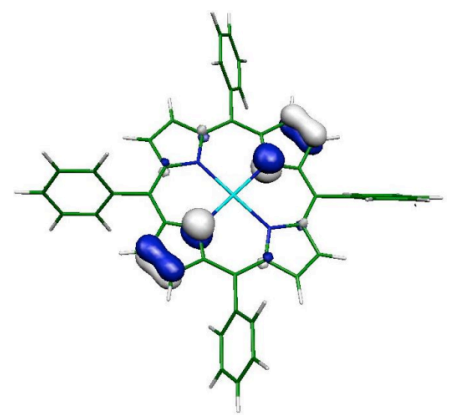

HOMO-4 (-6.49)

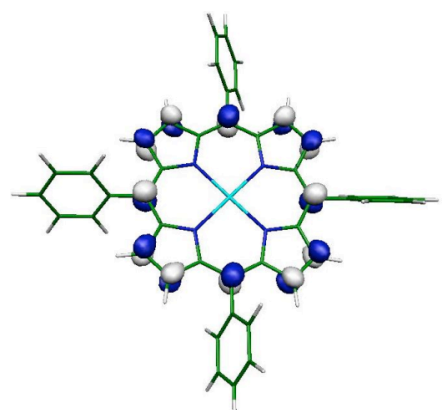

LUMO+2 (-0.66)

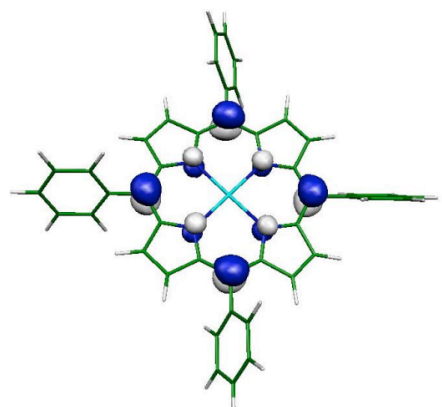

HOMO (-4.95)

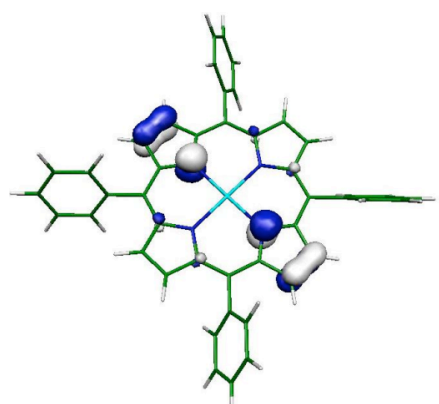

HOMO-3 (-6.49)

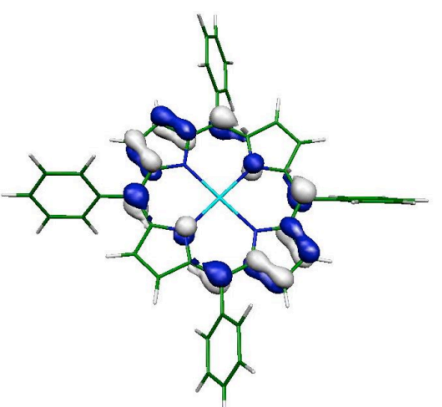

LUMO (-2.19)

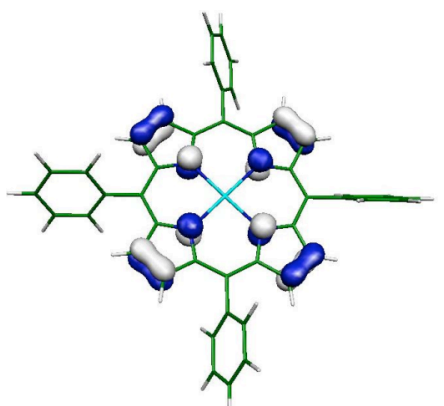

HOMO-2 (-6.46)

Figure S4. Molecular orbitals of TPPZn and their energies (in eV) calculated by the B3LYP method in toluene, where solvation effects were incorporated with the PCM method. 


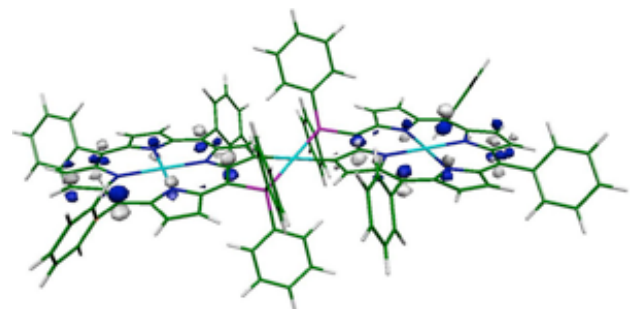

LUMO+3 (-1.93)

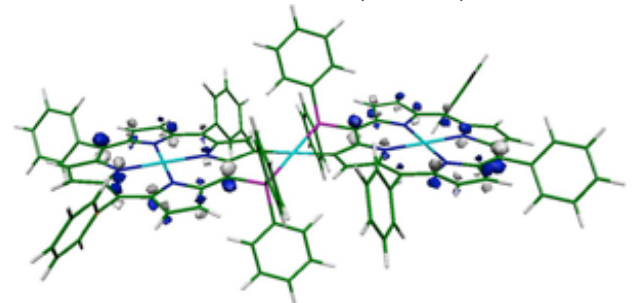

LUMO+1 (-2.14)

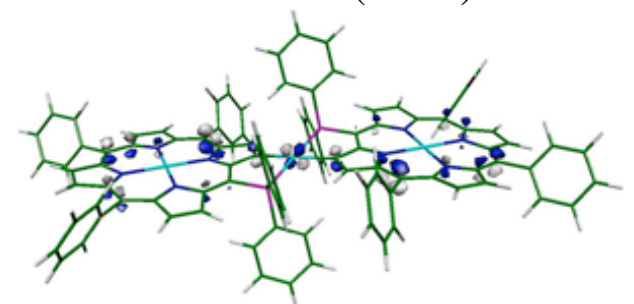

HOMO (-4.86)

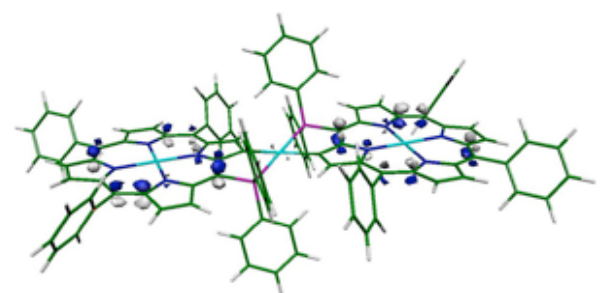

HOMO-2 (-4.97)
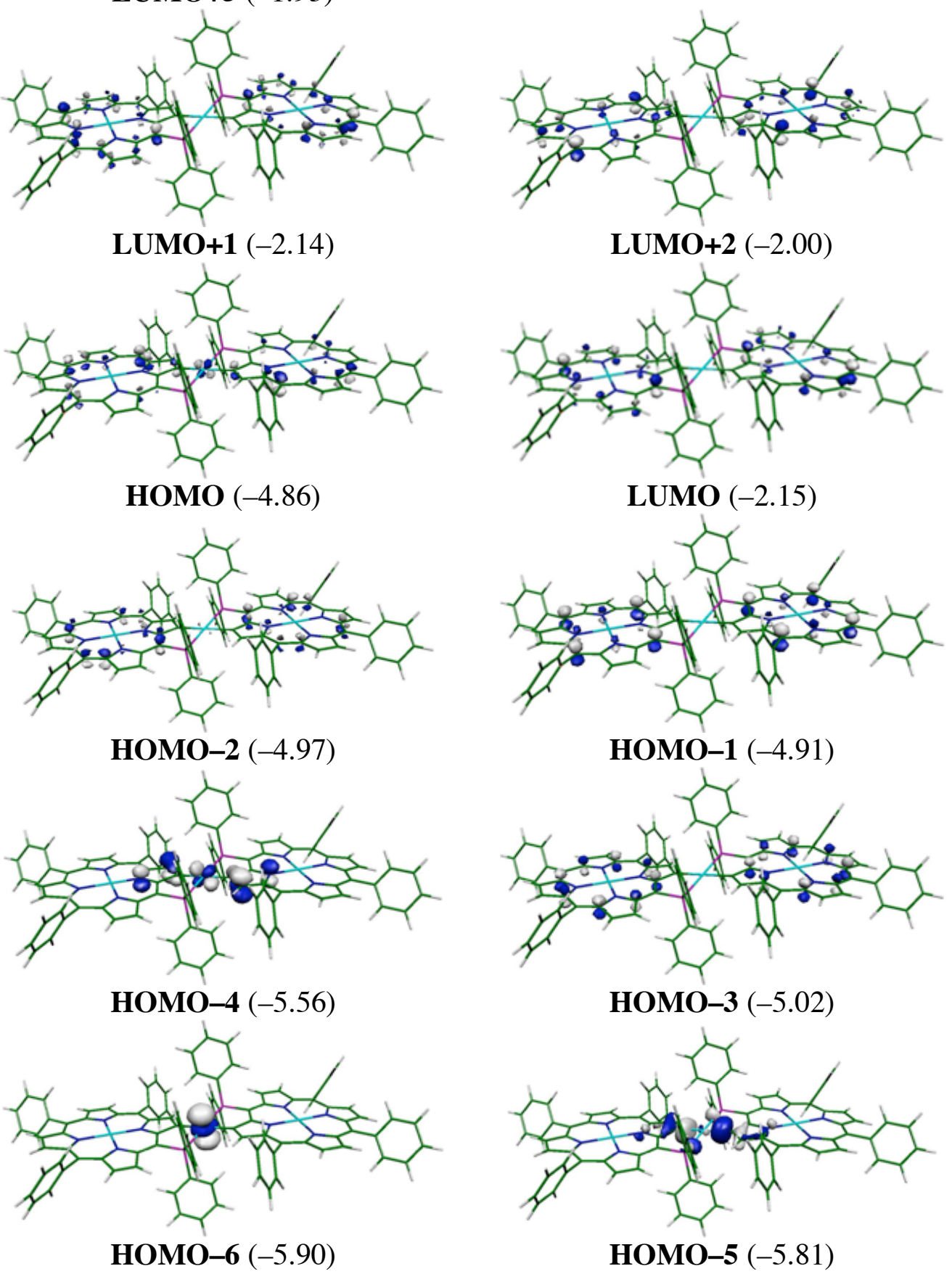

LUMO+2 (-2.00)

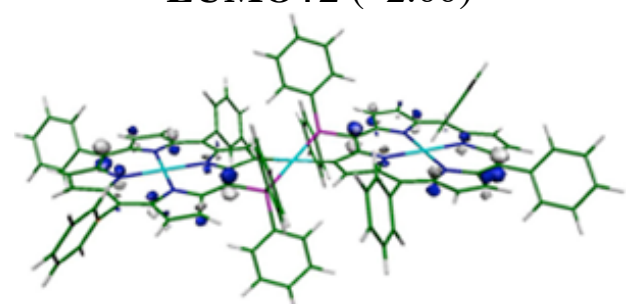

LUMO (-2.15)

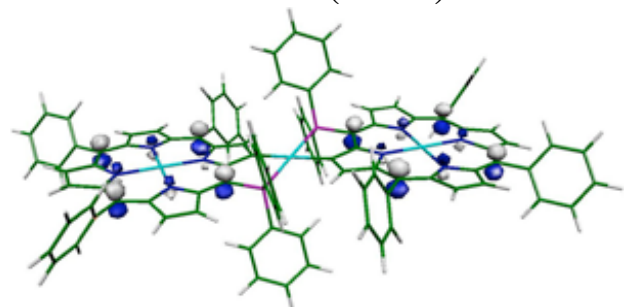

HOMO-1 (-4.91)

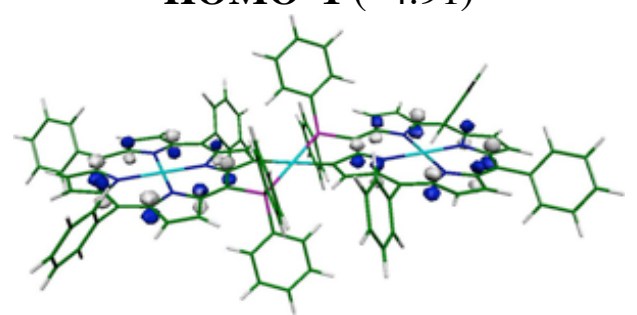

HOMO-3 (-5.02)

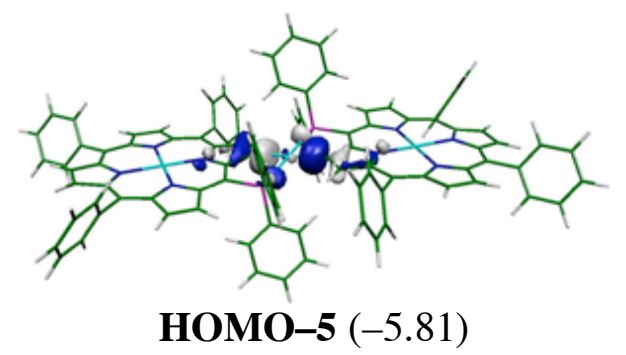

Figure S5. Molecular orbitals of $\mathbf{8}$ and their energies (in eV) calculated by the B3LYP method in gas phase. 

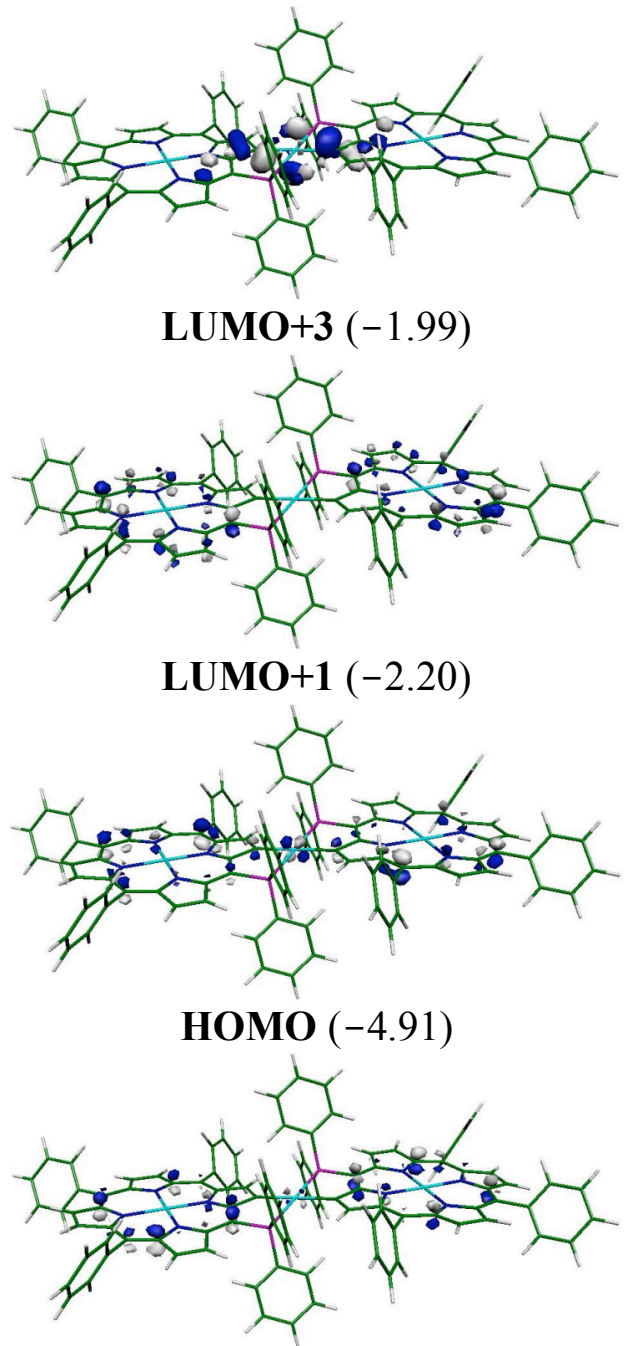

HOMO-2 (-5.03)
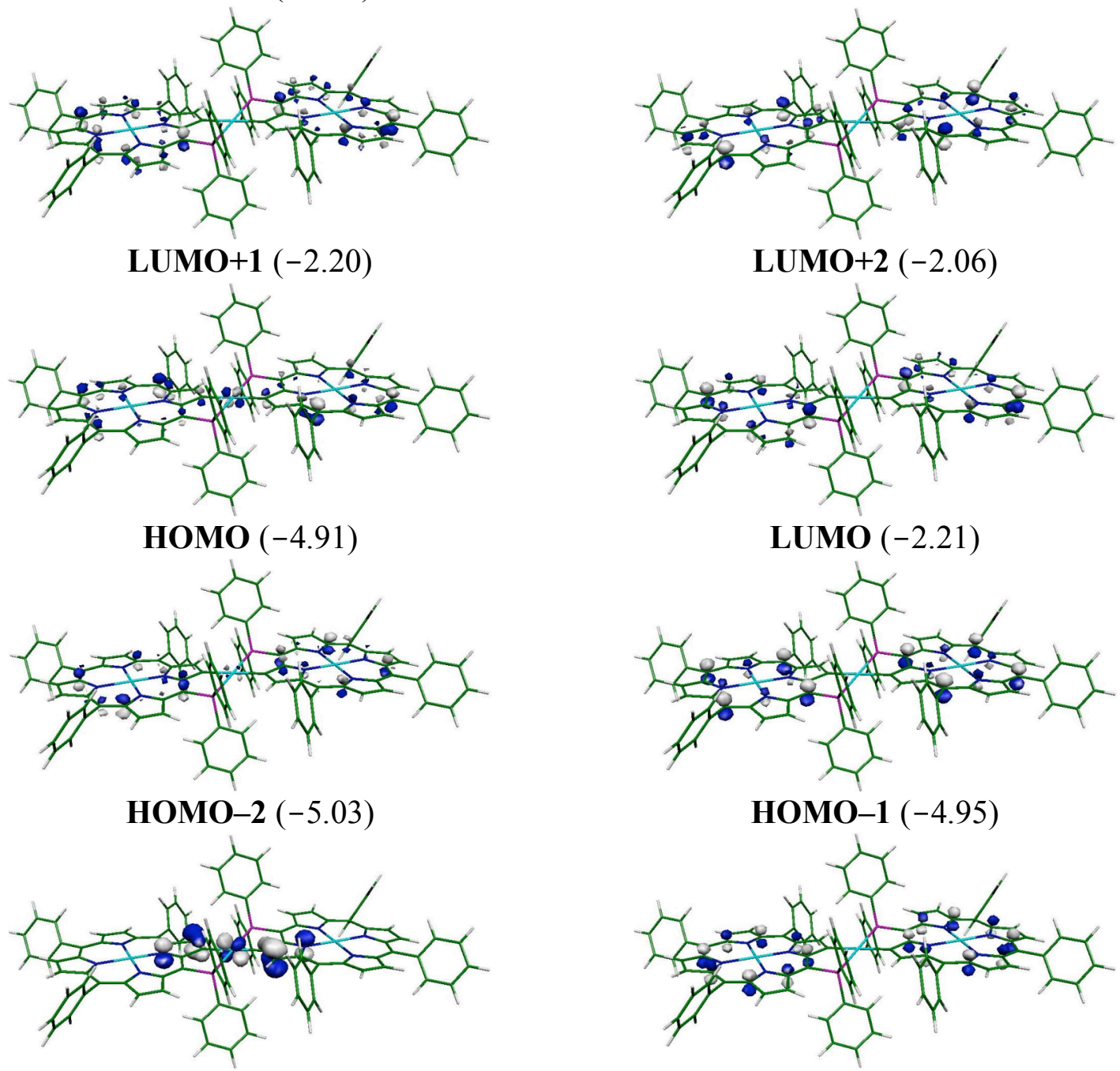

LUMO+2 (-2.06)

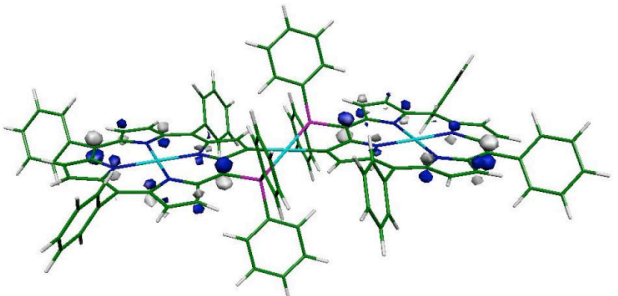

LUMO (-2.21)

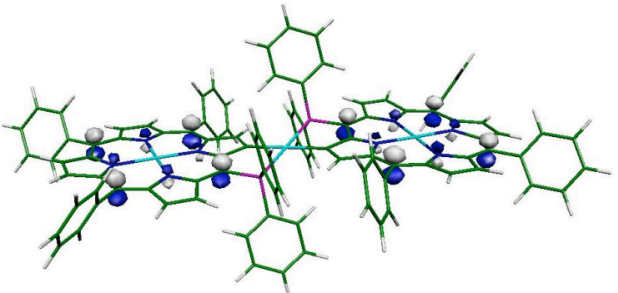

HOMO-1 (-4.95)

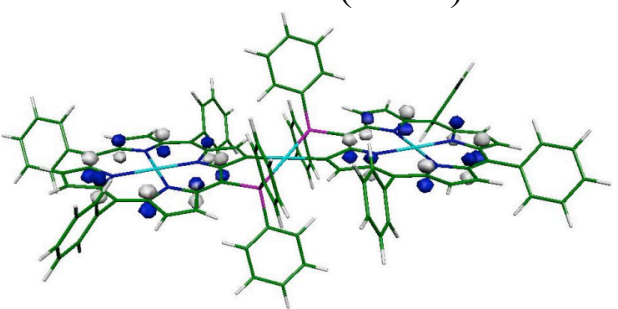

HOMO-4 (-5.62)
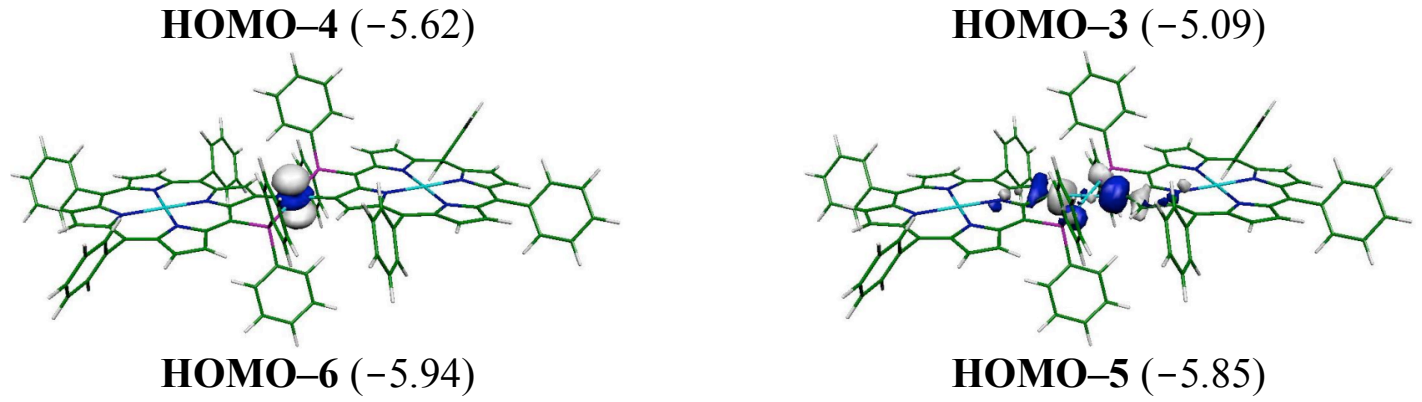

Figure S6. Molecular orbitals of 8 and their energies (in eV) calculated by the B3LYP method in toluene, where solvation effects were incorporated with the PCM method. 

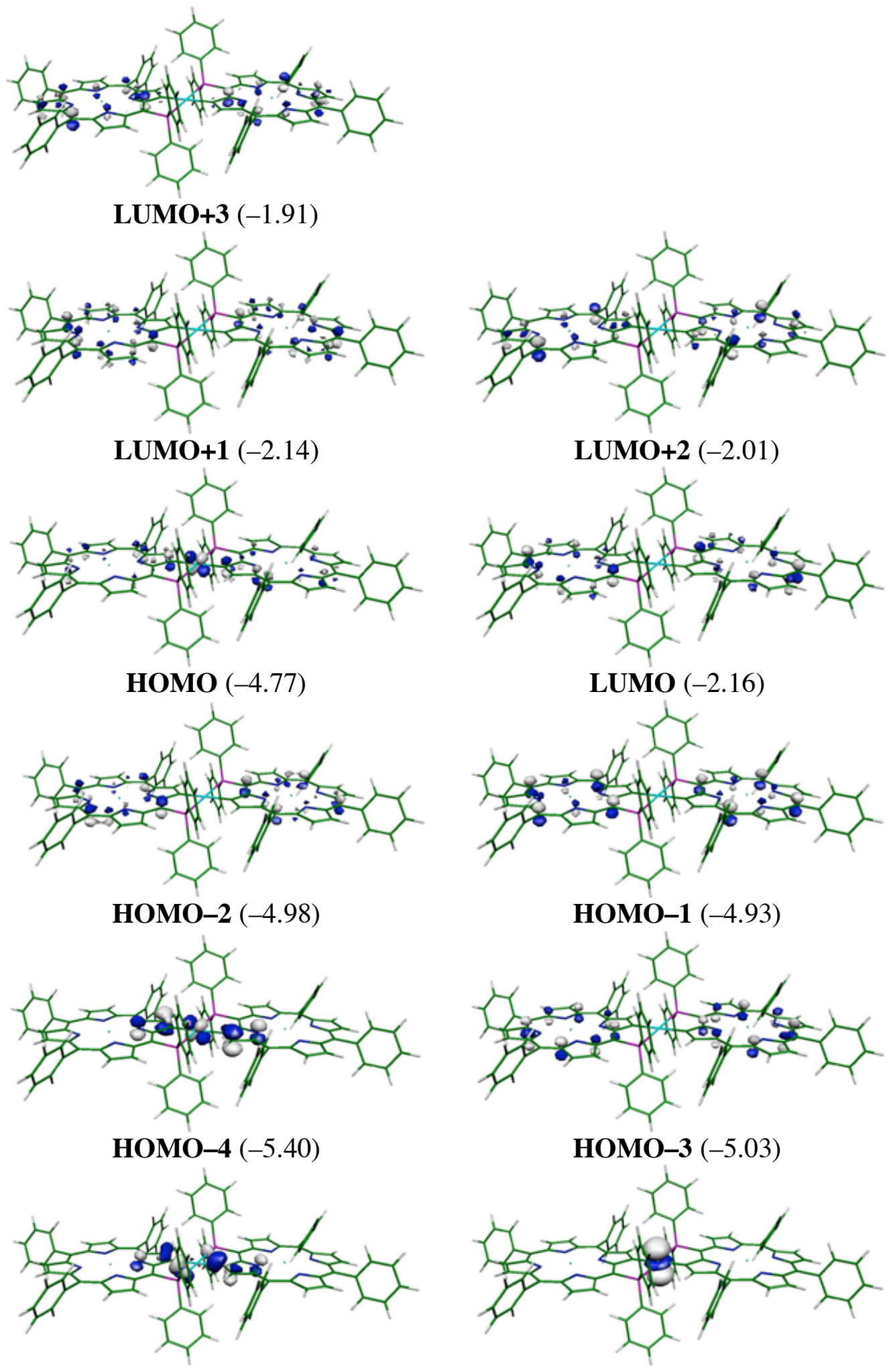

HOMO-6 (-5.96)
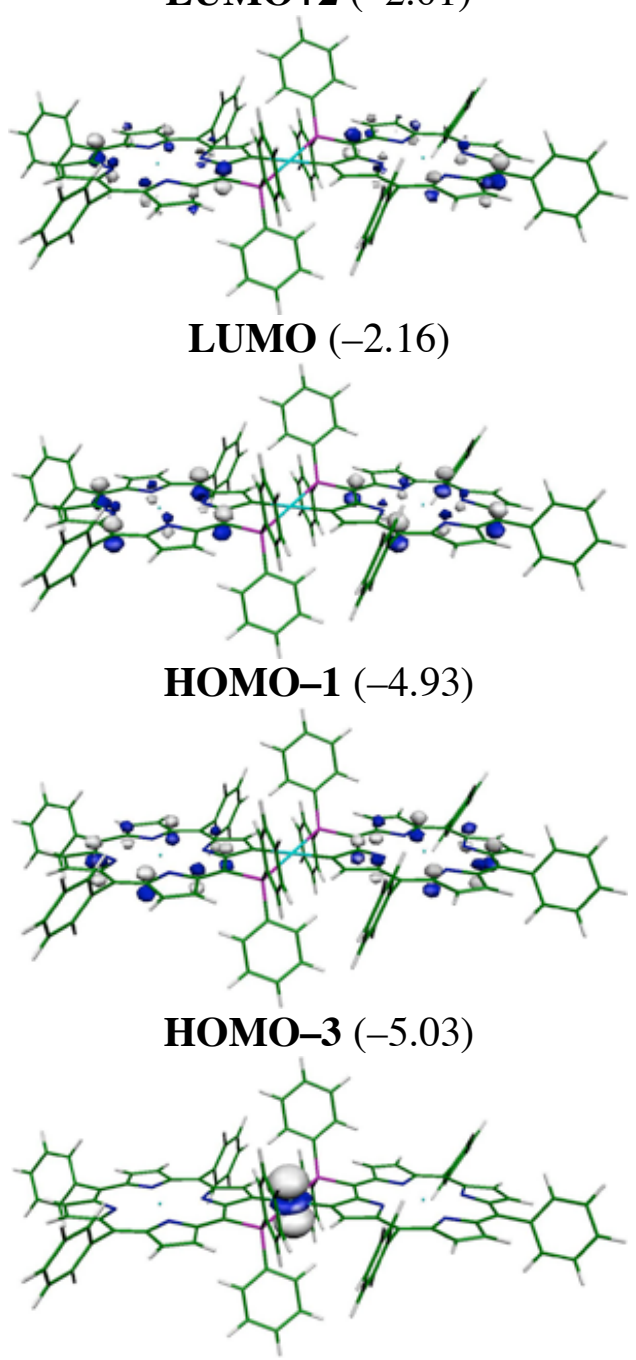

HOMO-5 (-5.88)

Figure S7. Molecular orbitals of 9 and their energies (in eV) calculated by the B3LYP method in gas phase. 

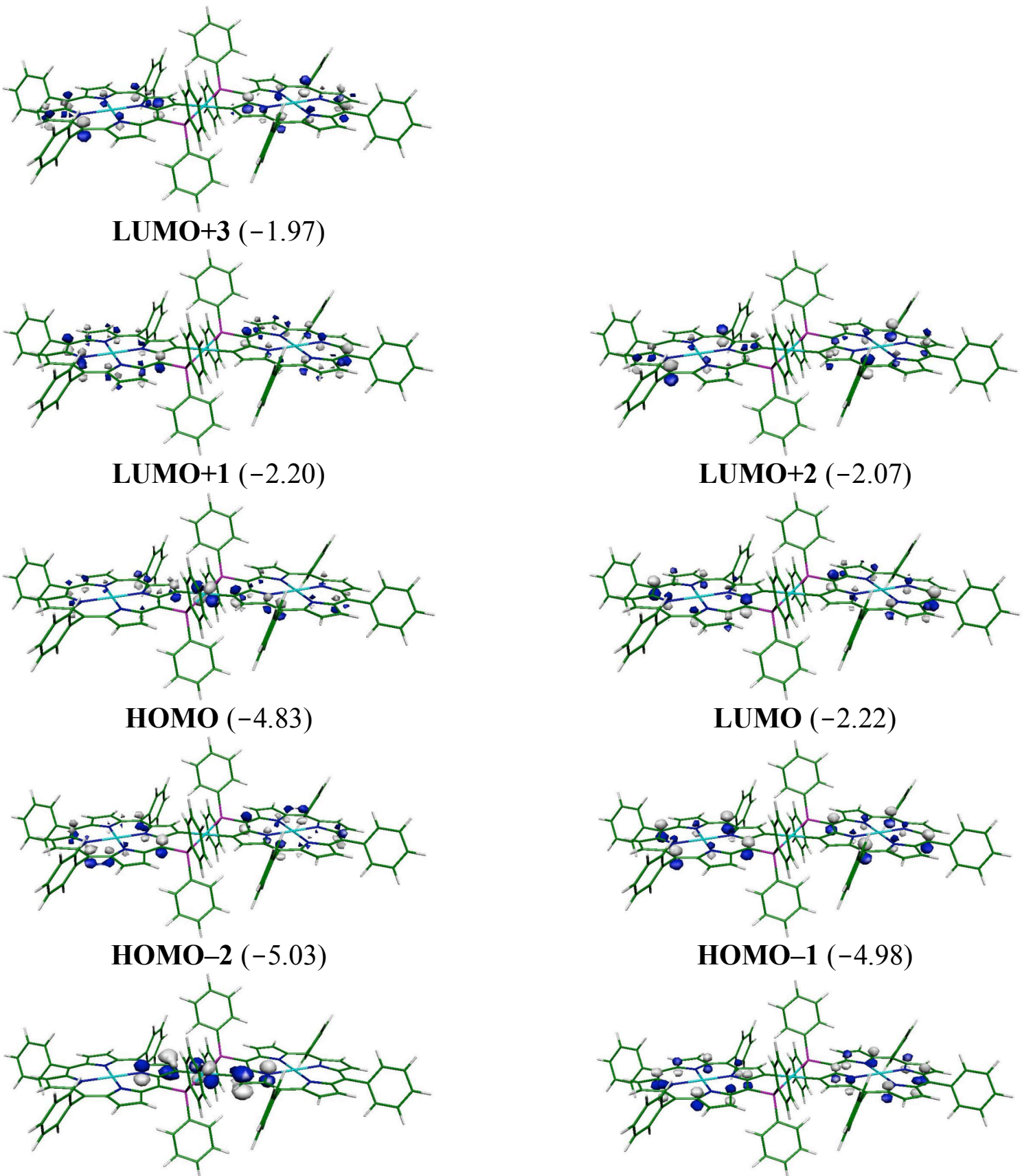

HOMO-1 (-4.98)
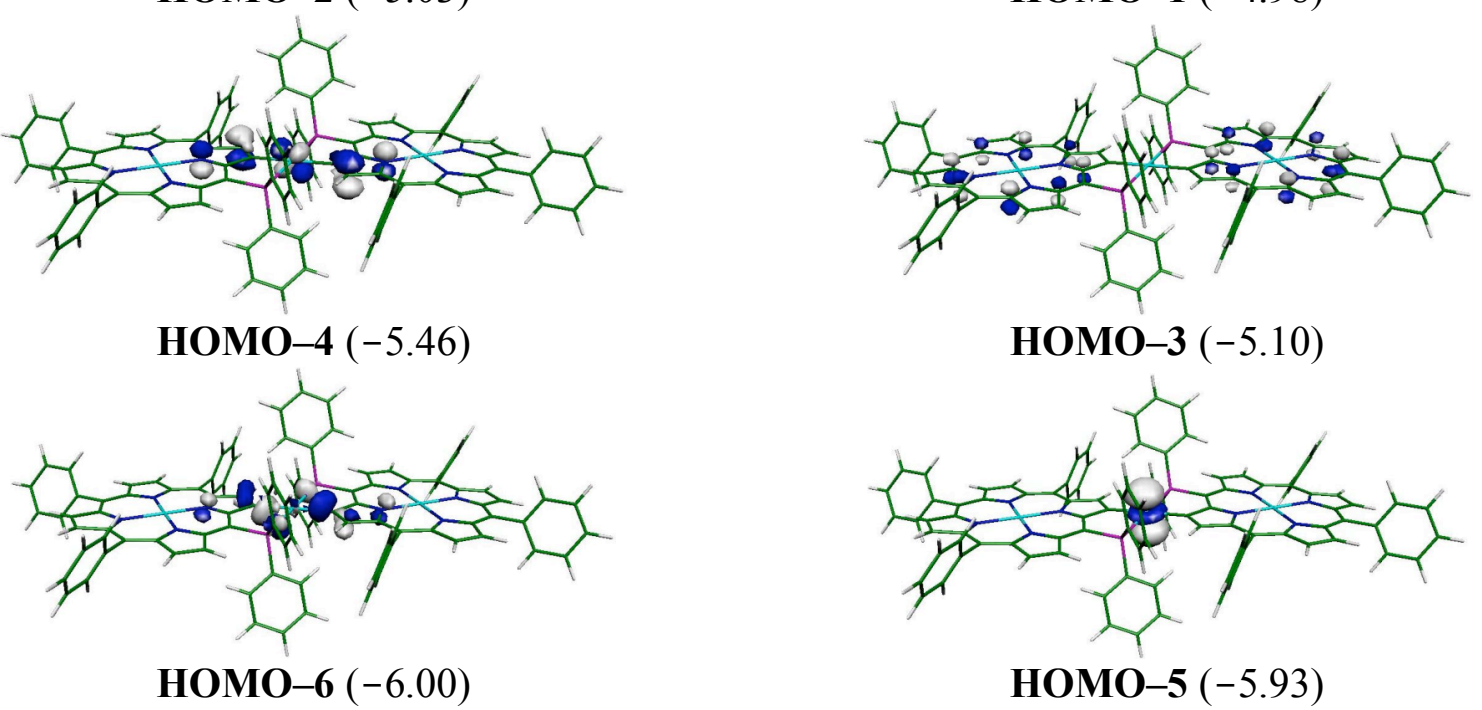

HOMO-3 (-5.10)

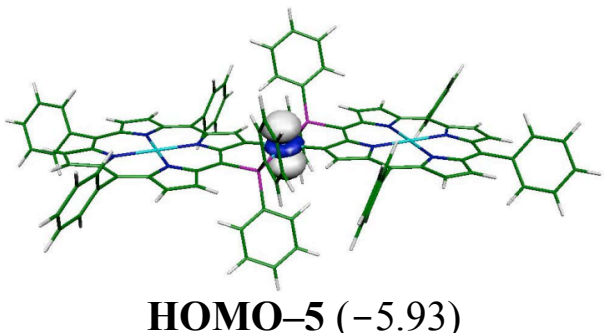

Figure S8. Molecular orbitals of 9 and their energies (in eV) calculated by the B3LYP method in toluene, where solvation effects were incorporated with the PCM method. 


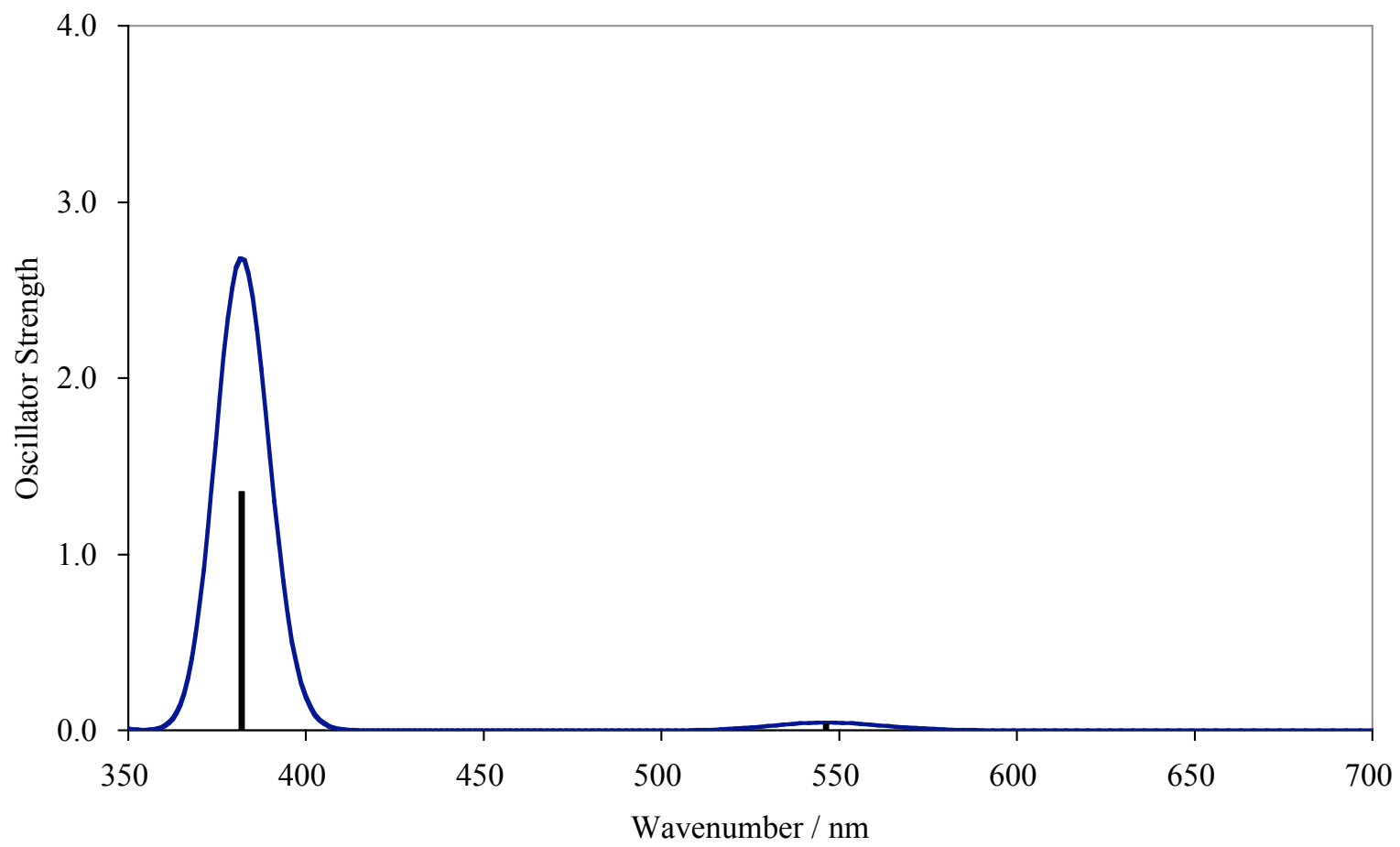

Figure S9. Absorption spectrum of TPPZn calculated by the TD-B3LYP method in gas phase.

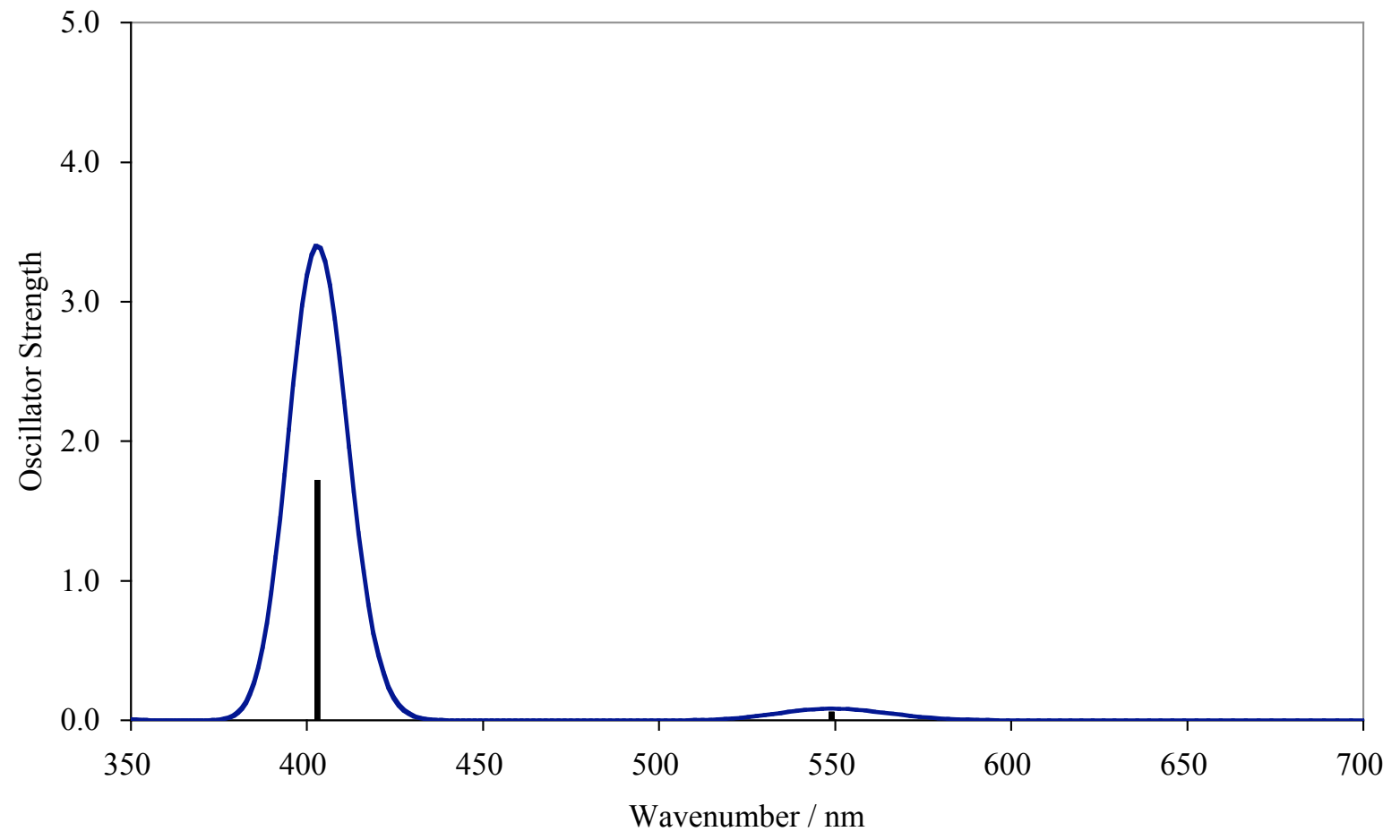

Figure S10. Absorption spectrum of TPPZn calculated by the TD-B3LYP method in toluene, where solvation effects were incorporated with the PCM method. 


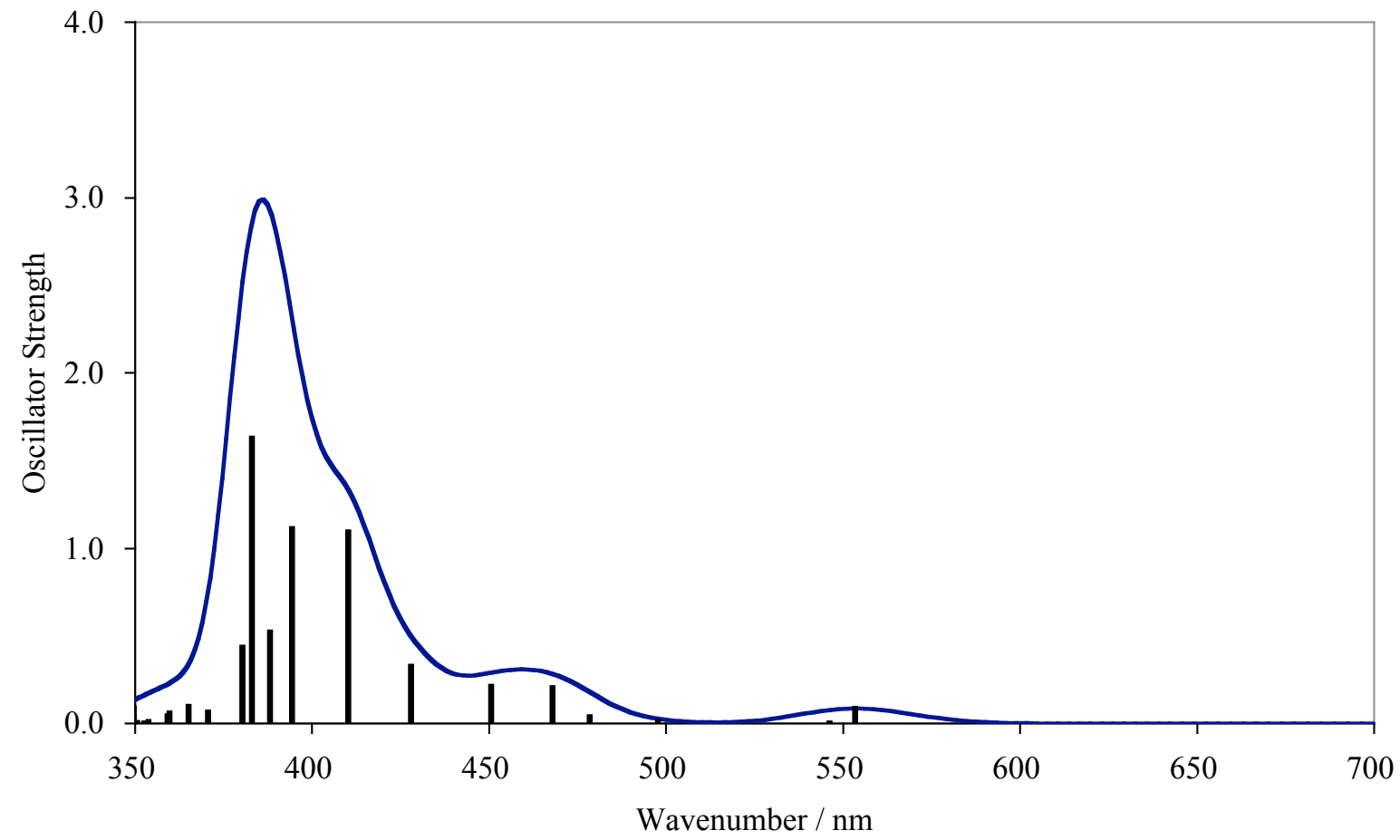

Figure S11. Absorption spectrum of $\mathbf{8}$ calculated by the TD-B3LYP method in gas phase.

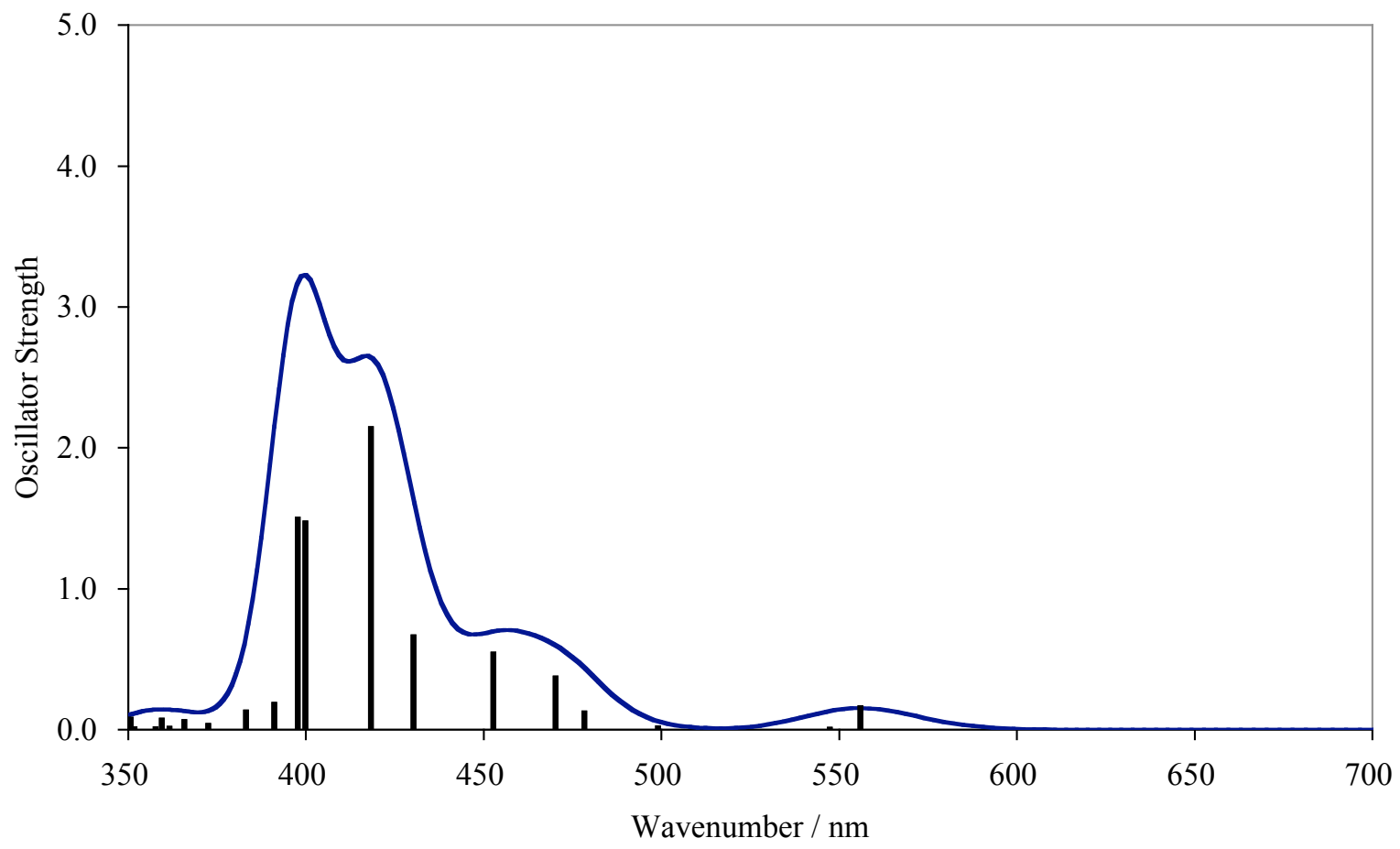

Figure S12. Absorption spectrum of $\mathbf{8}$ calculated by the TD-B3LYP method in toluene, where solvation effects were incorporated with the PCM method. 


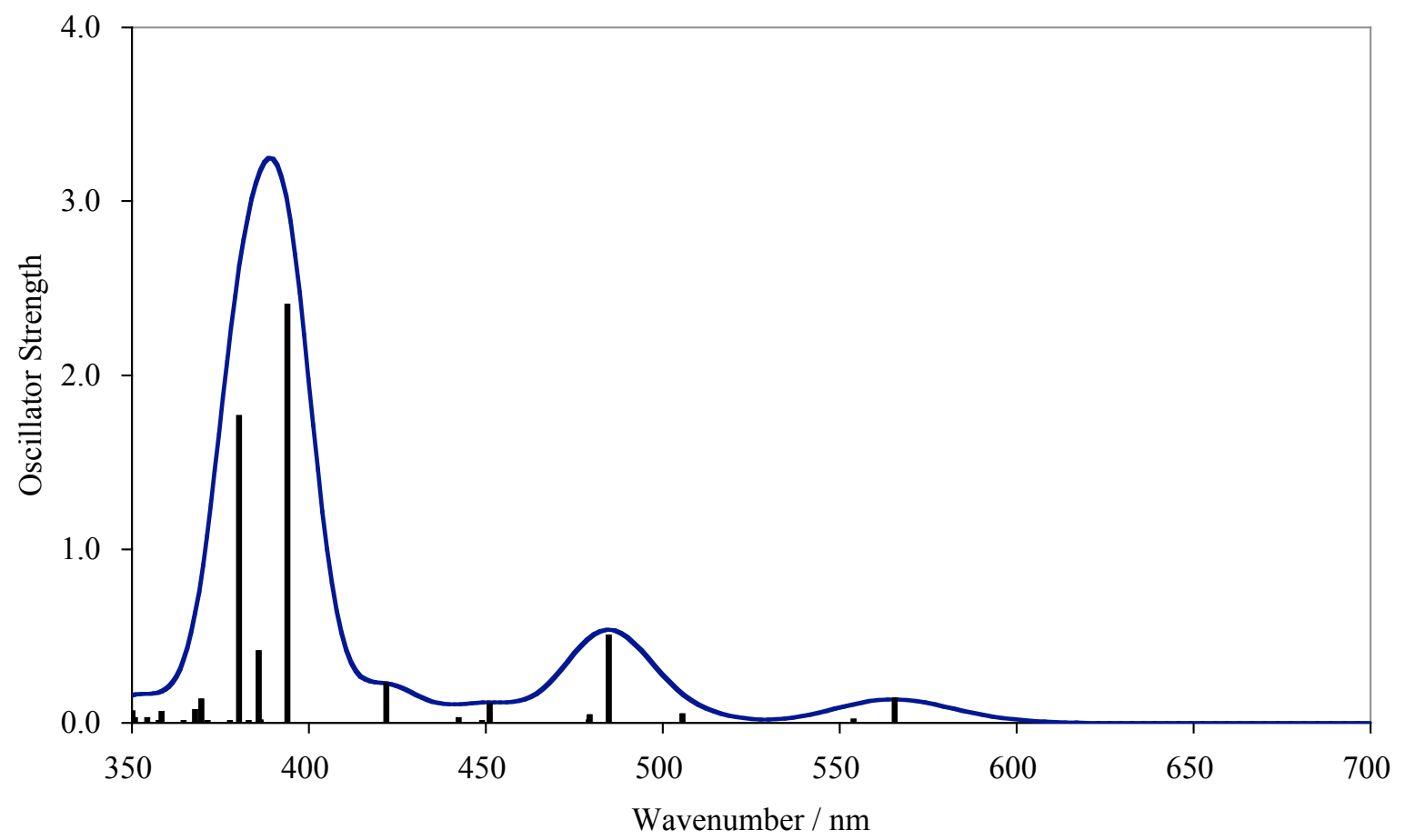

Figure S13. Absorption spectrum of $\mathbf{9}$ calculated by the TD-B3LYP method in gas phase.

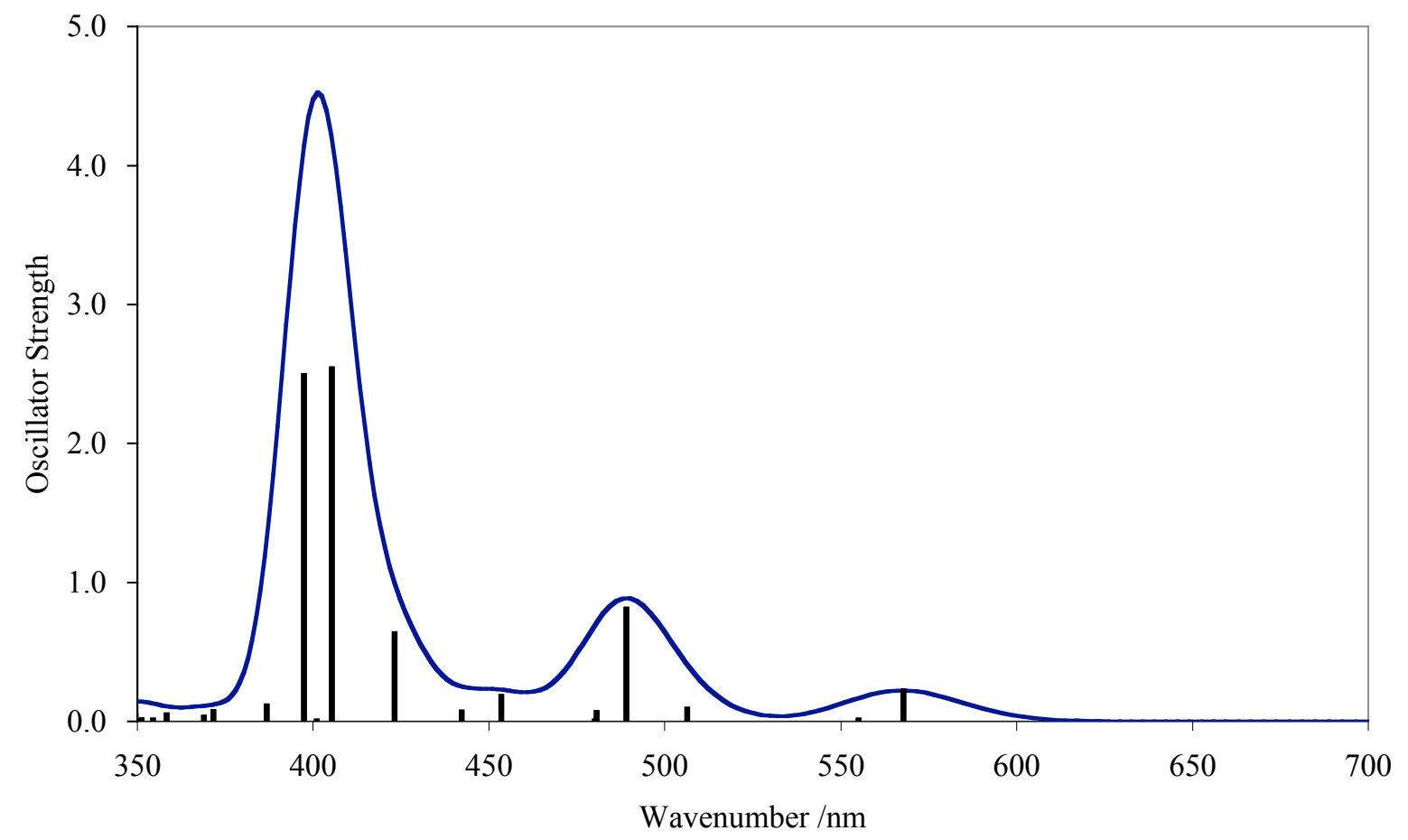

Figure S14. Absorption spectrum of 9 calculated by the TD-B3LYP method in toluene, where solvation effects were incorporated with the PCM method. 

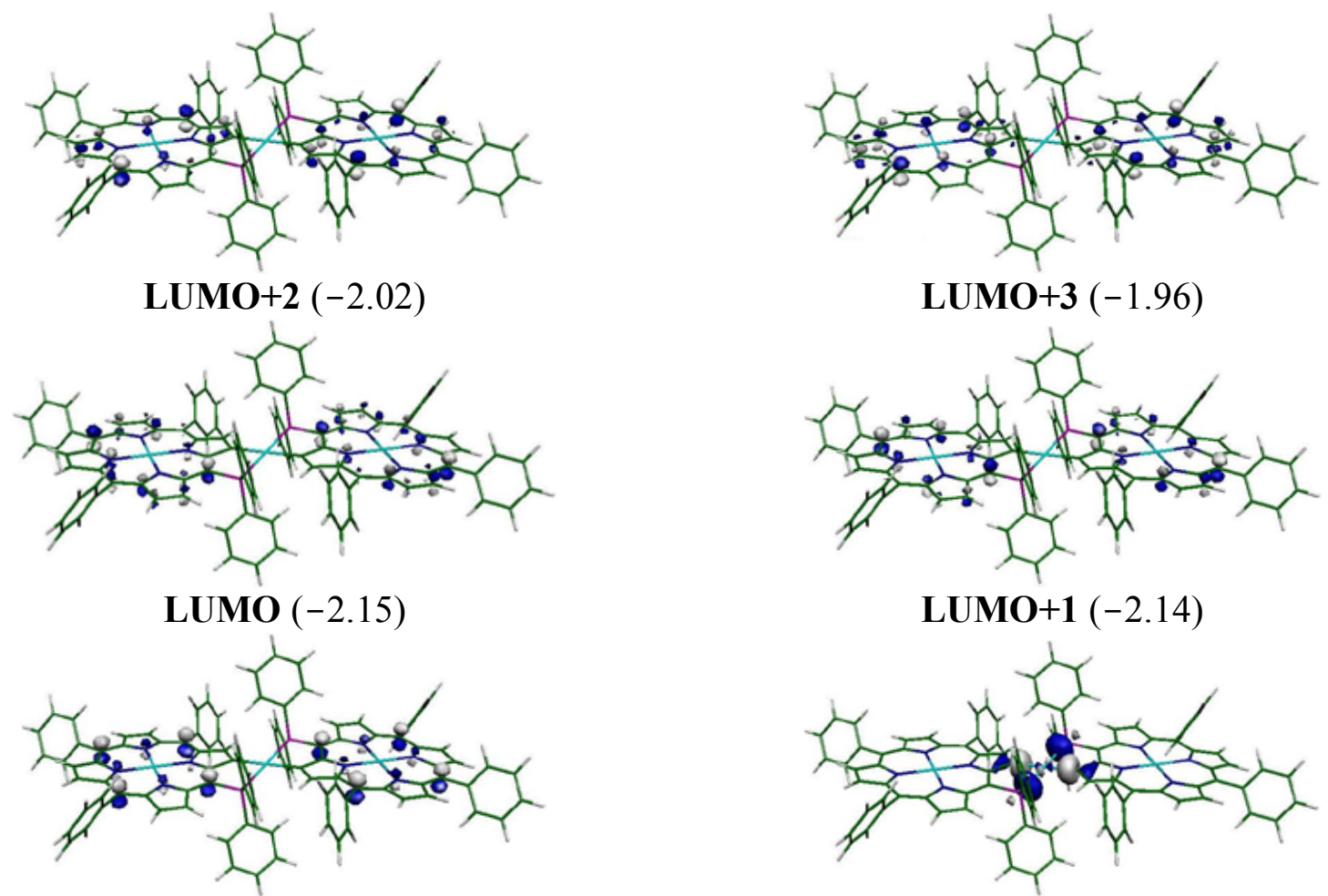

$$
\text { LUMO+1 (-2.14) }
$$
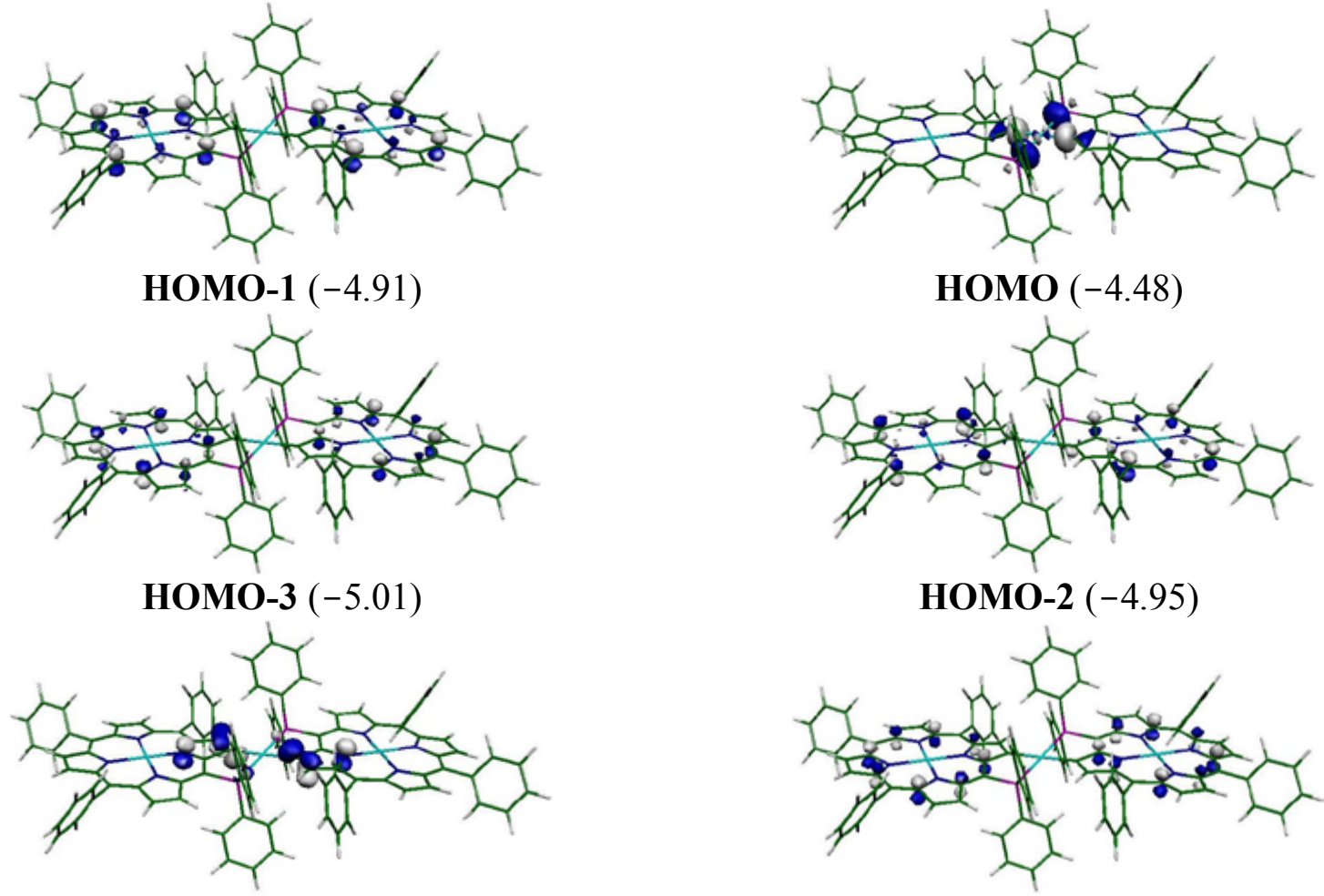

HOMO-2 (-4.95)
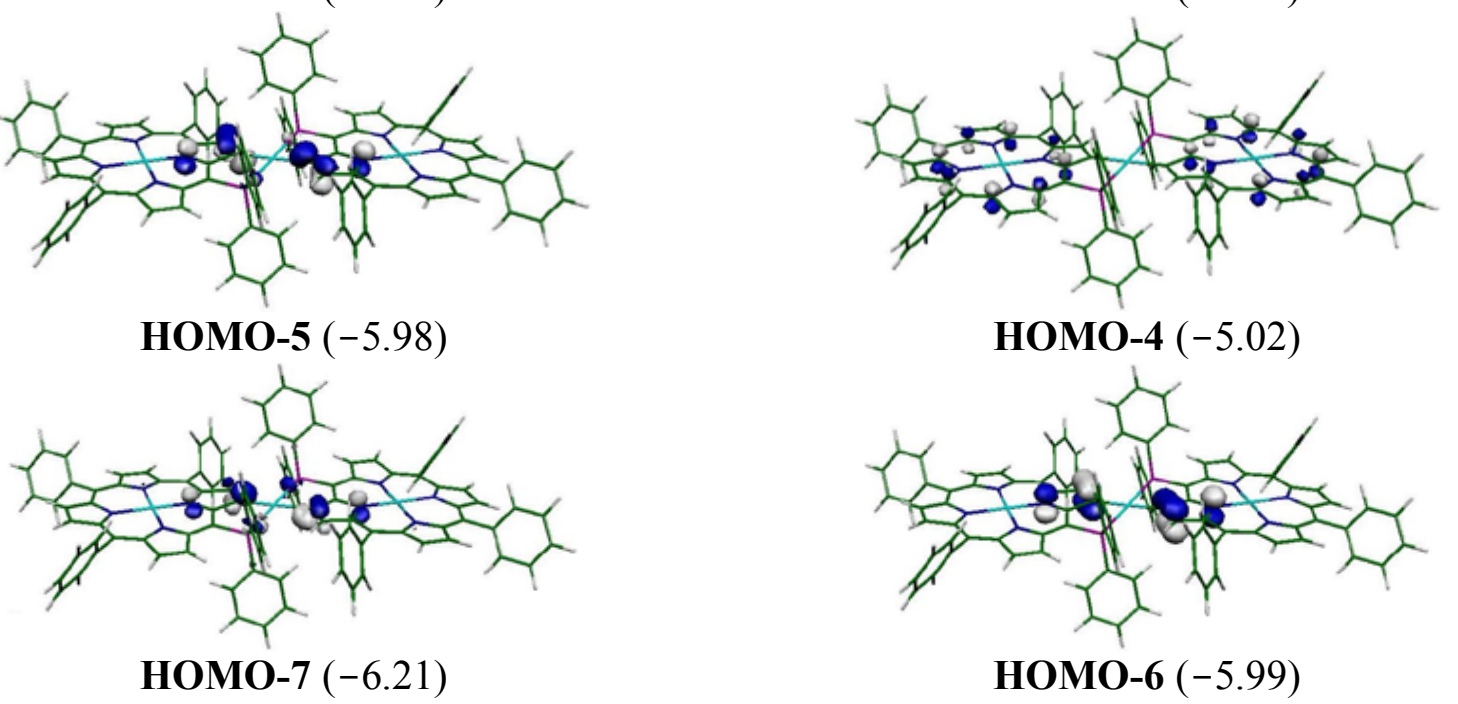

Figure S15. Molecular orbitals of Zn-ref and their energies (in eV) calculated by the B3LYP method in gas phase. 

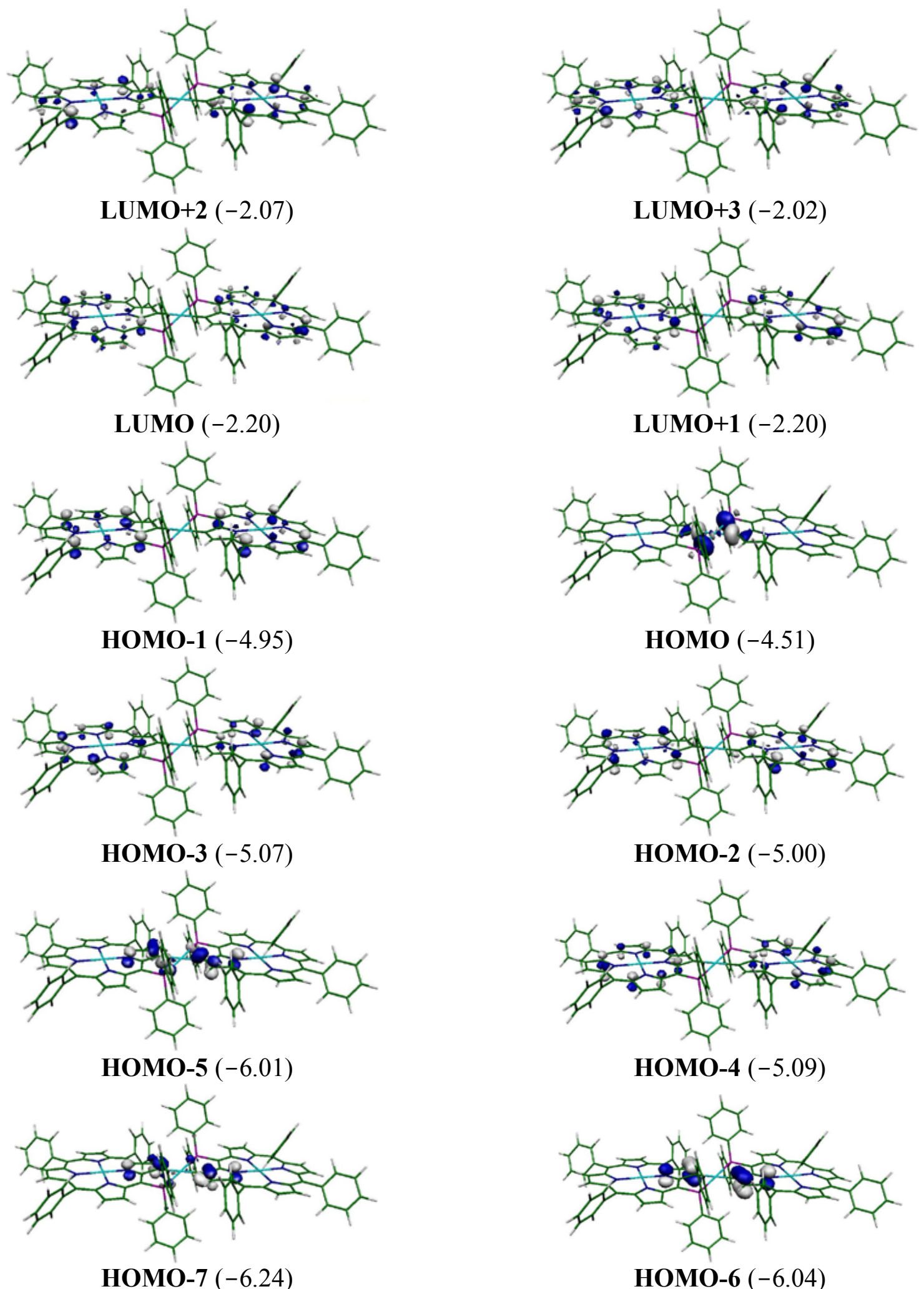

HOMO-4 (-5.09)

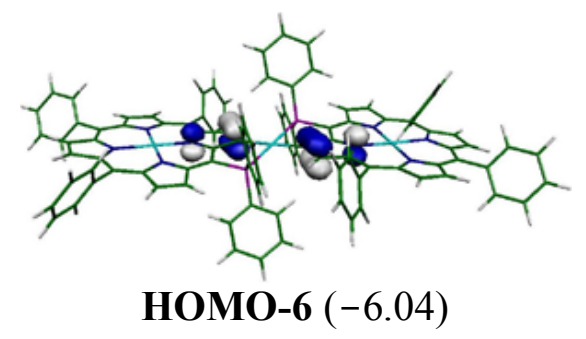

Figure S16. Molecular orbitals of Zn-ref and their energies (in eV) calculated by the B3LYP method in toluene, where solvation effects were incorporated with the PCM method. 


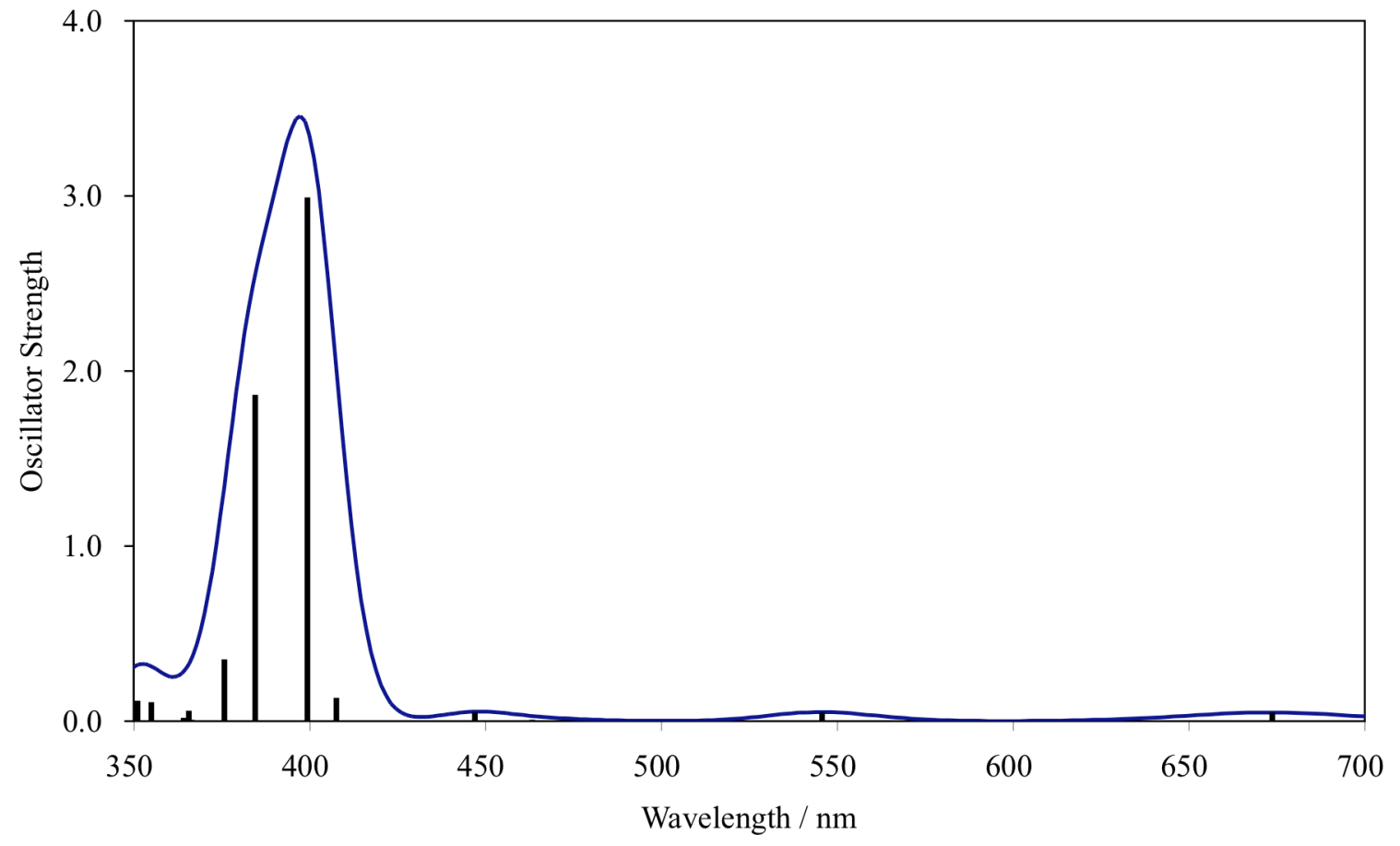

Figure S17. Absorption spectrum of Zn-ref calculated by the TD-B3LYP method in gas phase. The identical structure of $\mathbf{8}$ optimized by the B3LYP method was used.

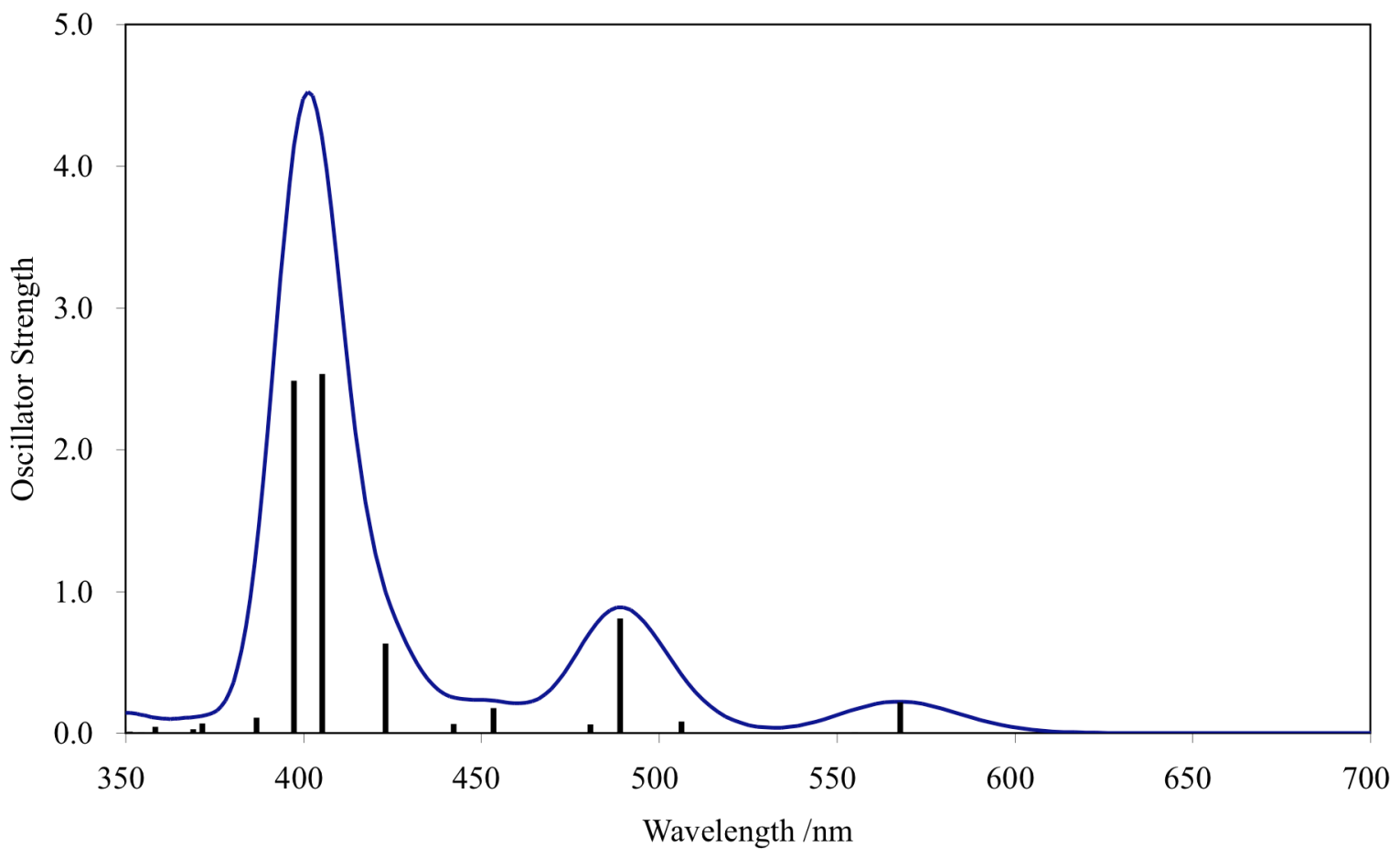

Figure S18. Absorption spectrum of Zn-ref calculated by the TD-B3LYP method in toluene, where solvation effects were incorporated with the PCM method. The identical structure of 8 optimized by the B3LYP method was used. 
(a)

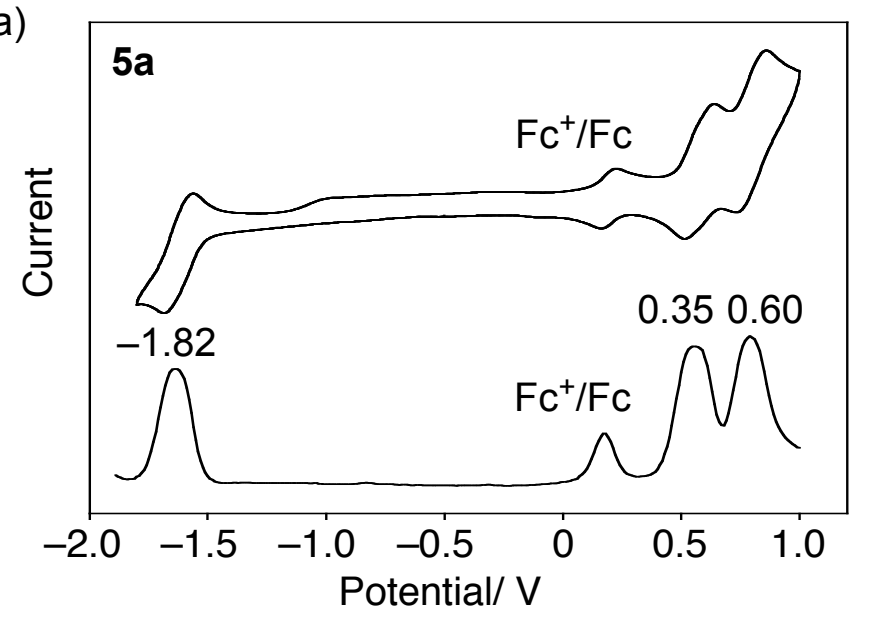

(b)

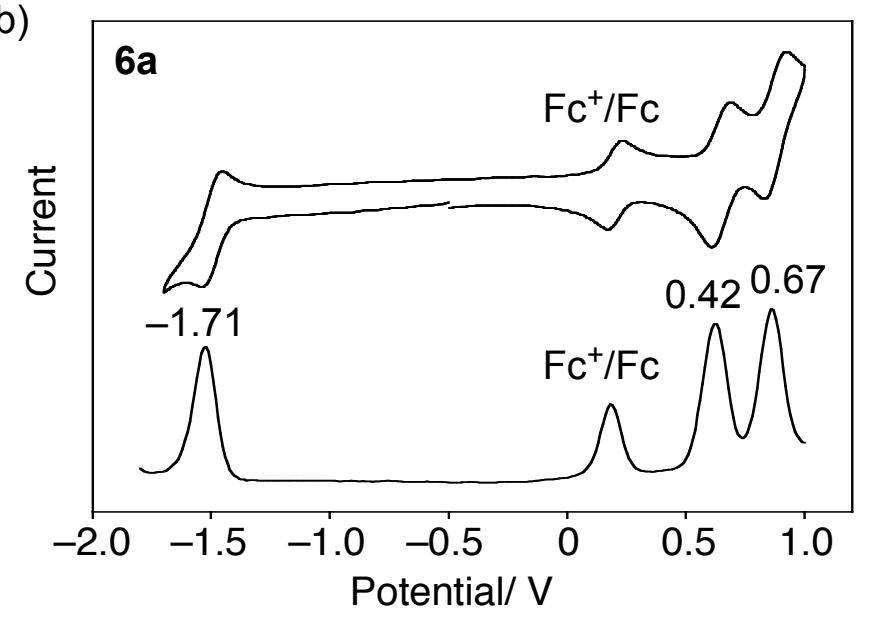

(c)

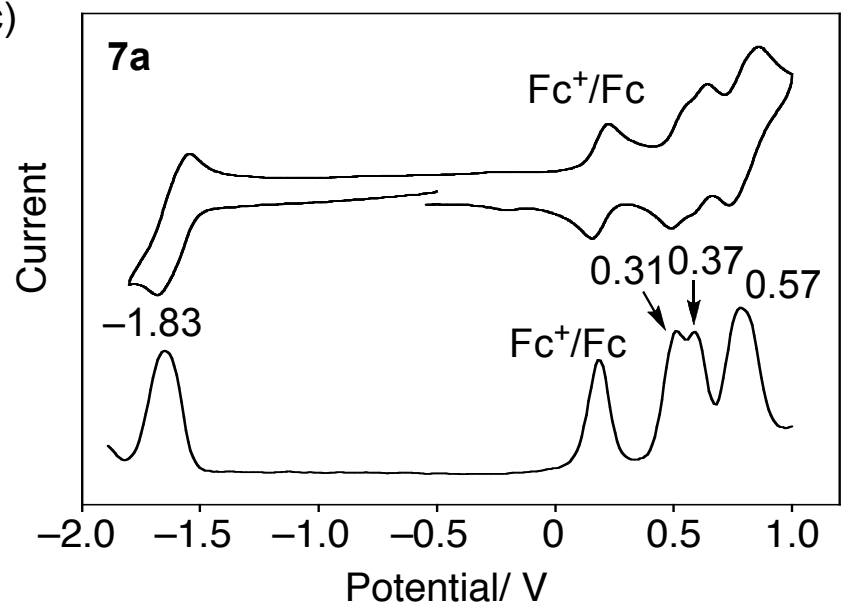

Figure S19. Cyclic voltammograms (upper) and differential pulse voltammograms (lower) in the range

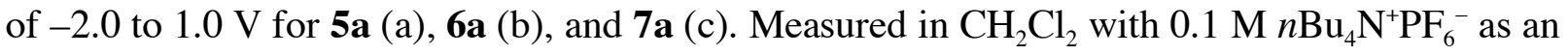
electrolyte; $\mathrm{Ag} / \mathrm{Ag}^{+}\left[\mathrm{AgNO}_{3}(\mathrm{MeCN})\right]$ as a reference electrode; Scan rate $20 \mathrm{mV} \mathrm{s}^{-1}$. Redox potentials (in $\mathrm{V}$ ) relative to that of $\mathrm{Fc}^{+} / \mathrm{Fc}$ are shown above DPV voltammograms. 


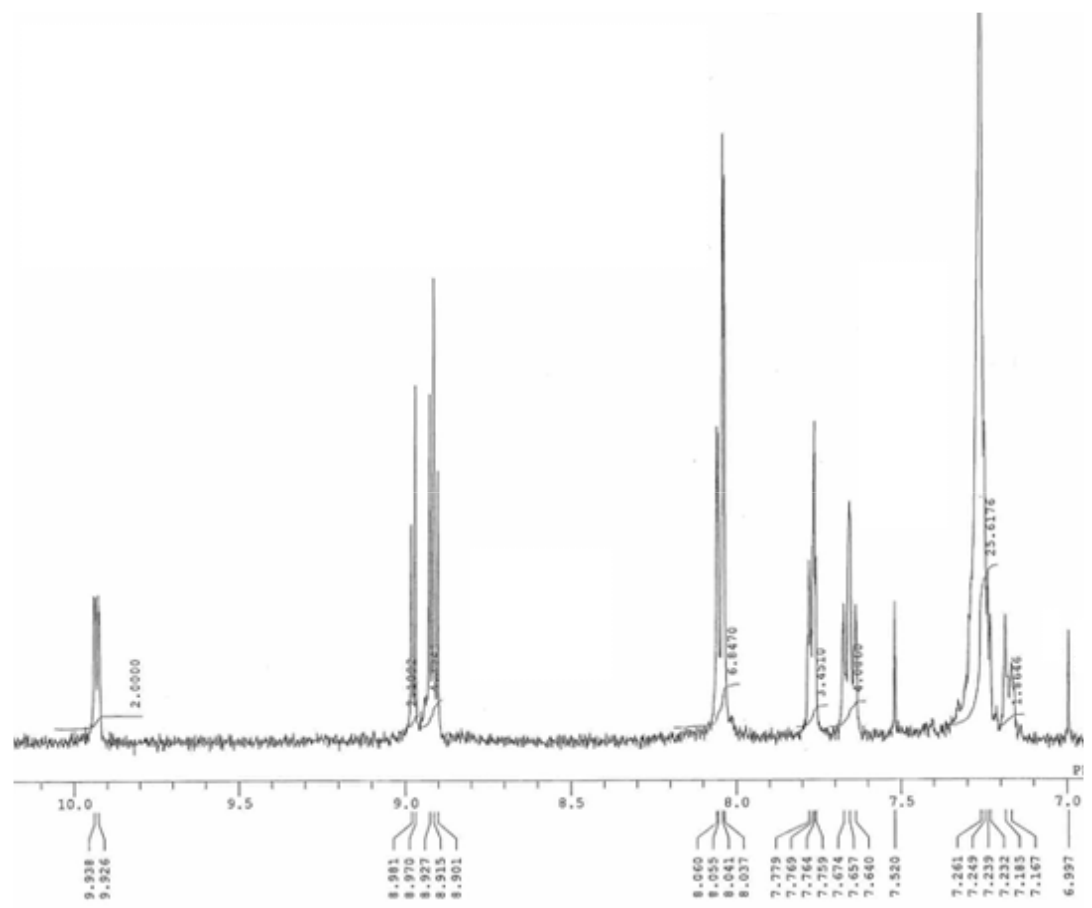

Figure S20. ${ }^{1} \mathrm{H}$ NMR spectrum (aromatic region) of $\mathbf{2 a}$ in $\mathrm{CDCl}_{3}$.

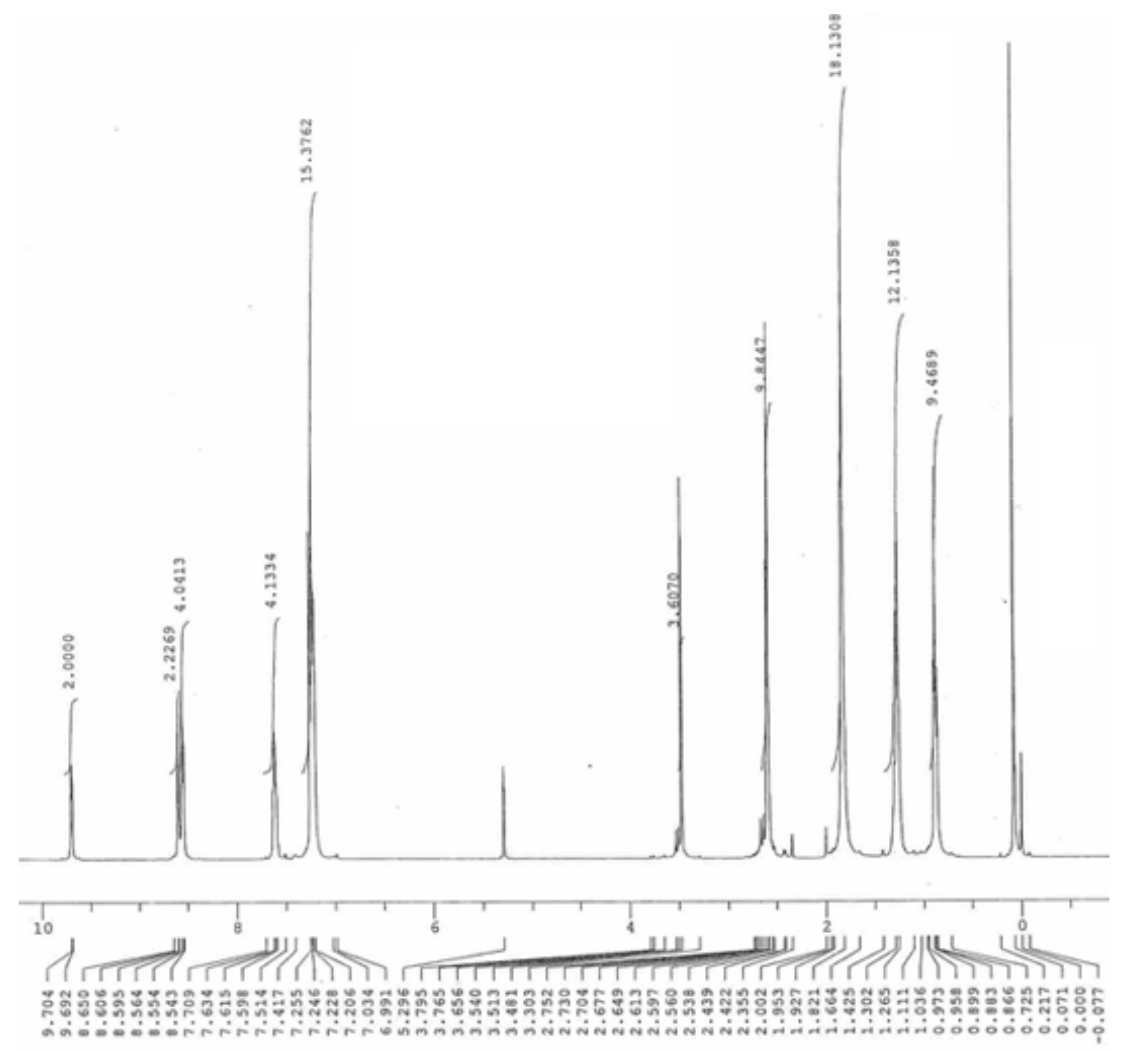

Figure S21. ${ }^{1} \mathrm{H}$ NMR spectrum of $\mathbf{2 b}$ in $\mathrm{CDCl}_{3}$.

SI- 28 


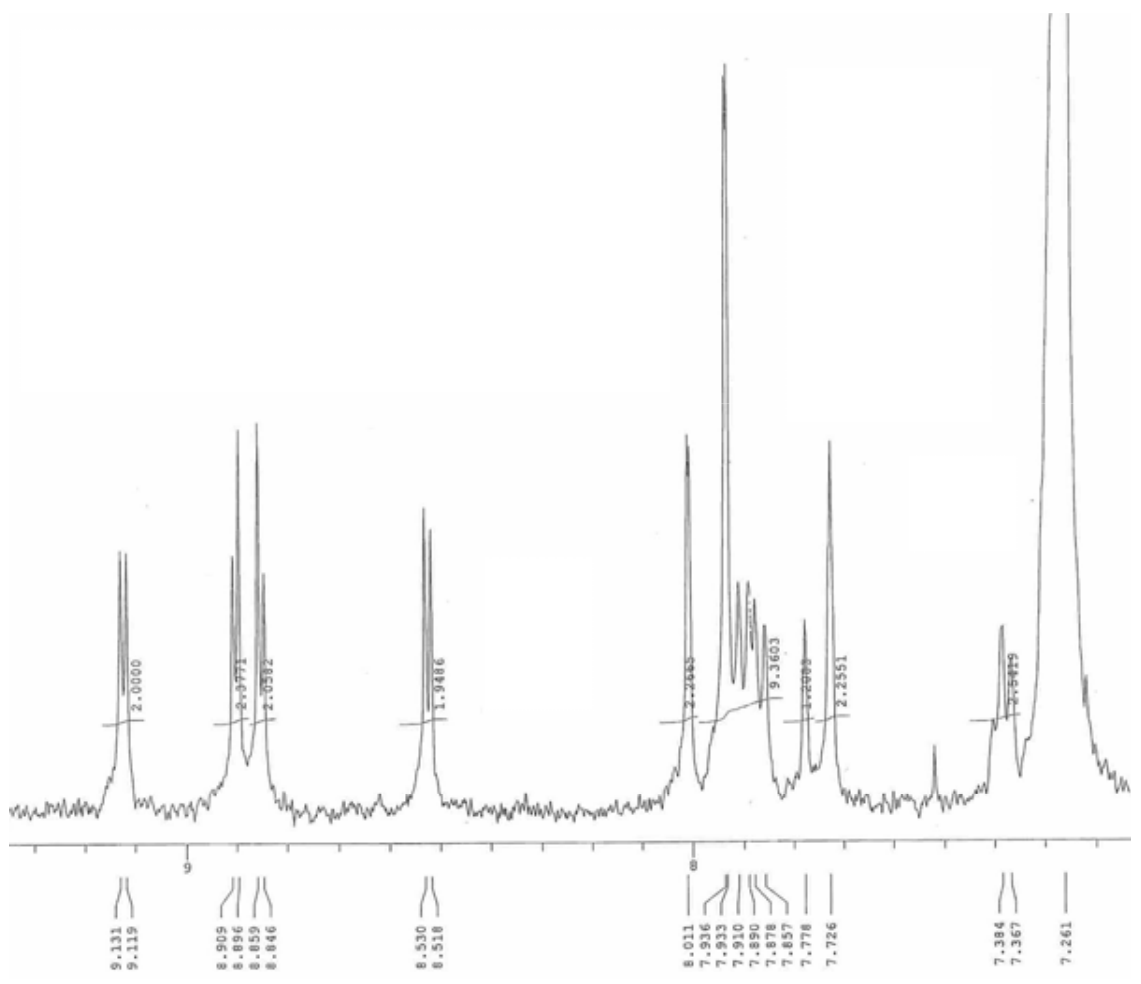

Figure S22. ${ }^{1} \mathrm{H}$ NMR spectrum (aromatic region) of $\mathbf{4 a}$ in $\mathrm{CDCl}_{3}$.

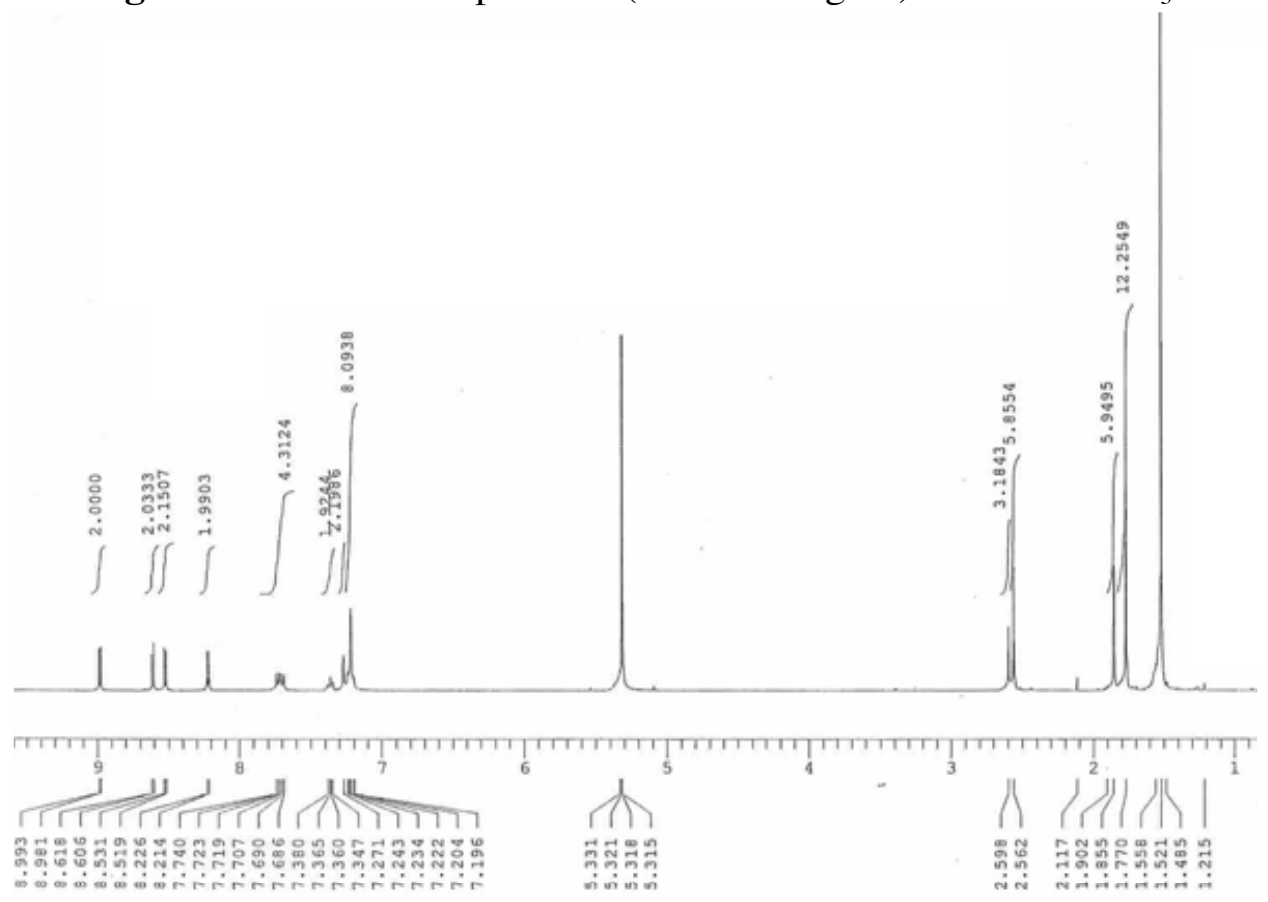

Figure S23. ${ }^{1} \mathrm{H}$ NMR spectrum of $\mathbf{4 b}$ in $\mathrm{CD}_{2} \mathrm{Cl}_{2}$. 


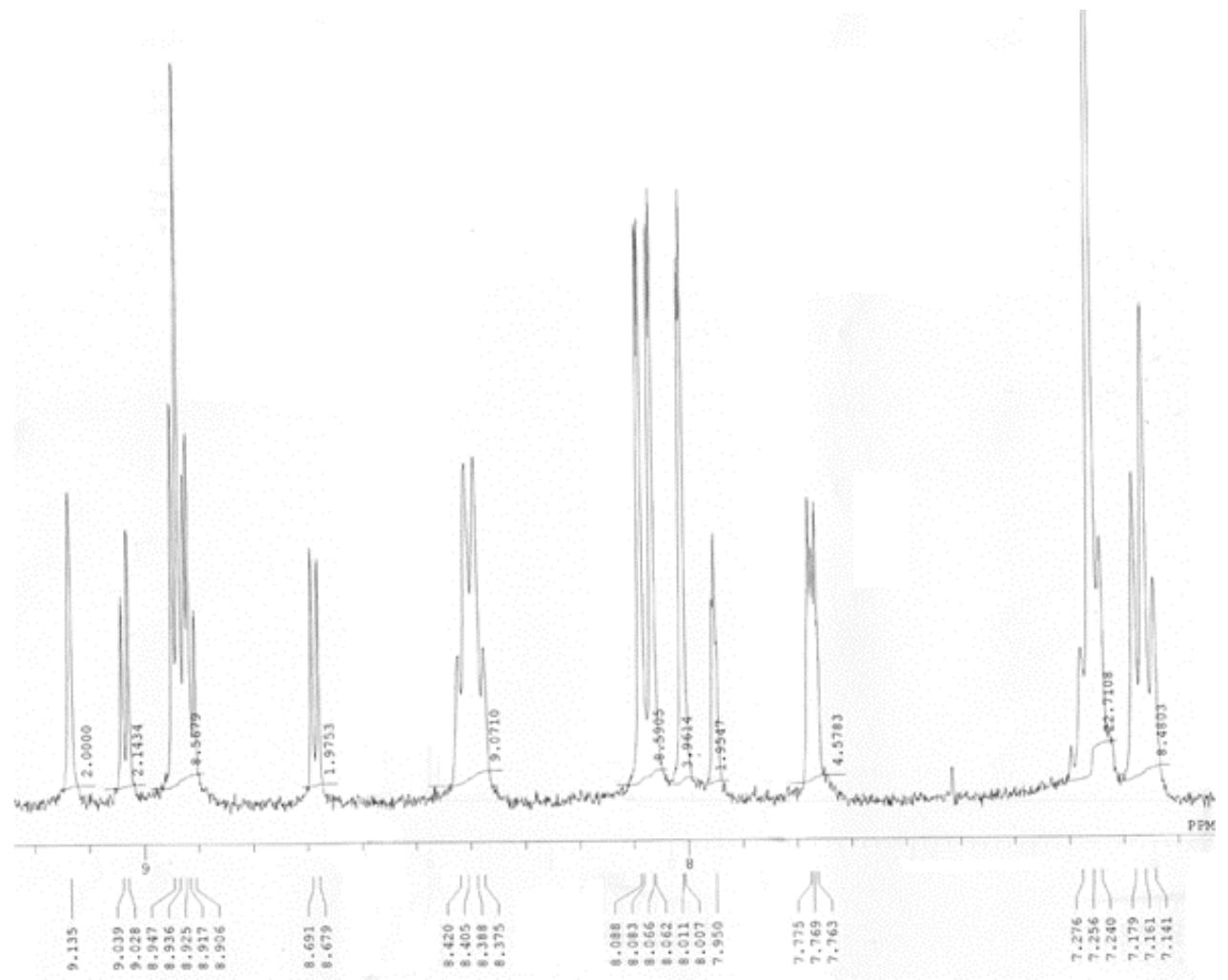

Figure S24. ${ }^{1} \mathrm{H}$ NMR spectrum (aromatic region) of $\mathbf{5 a}$ in $\mathrm{CDCl}_{3}$.

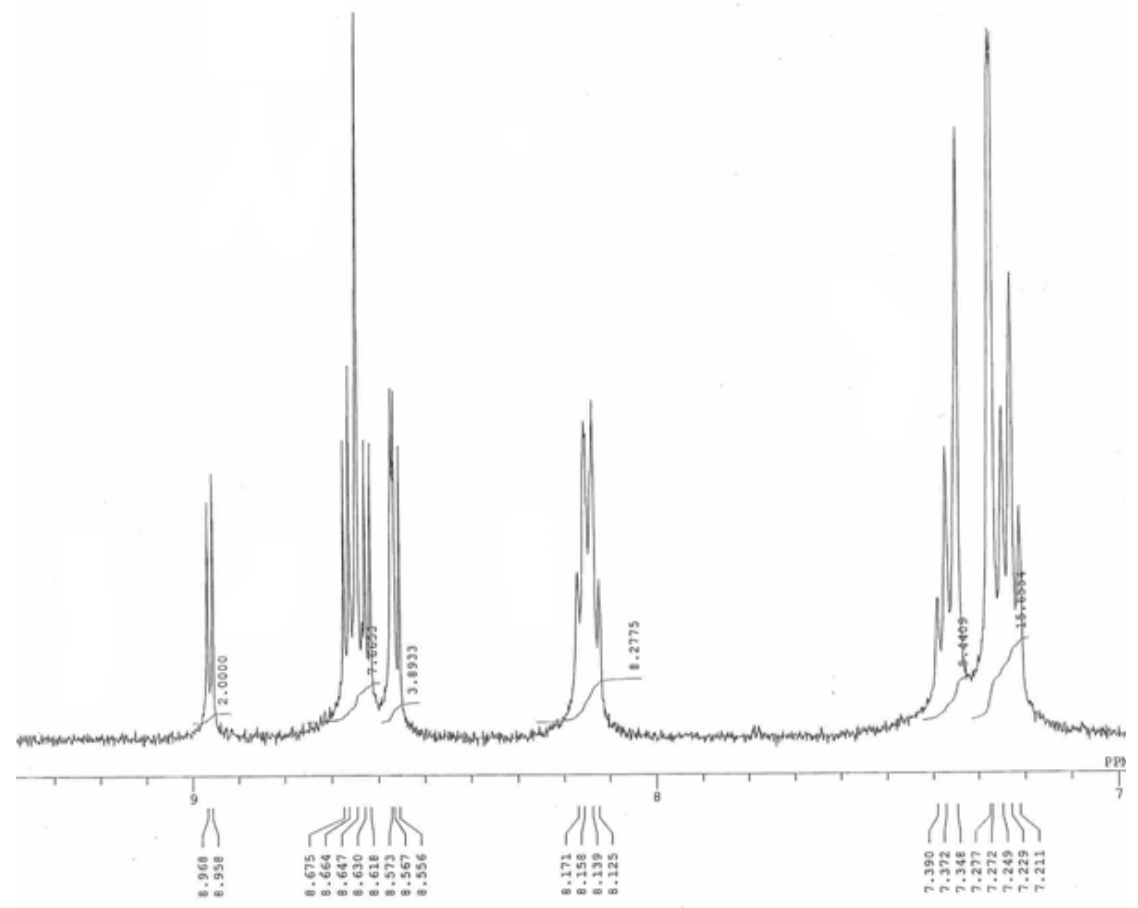

Figure S25. ${ }^{1} \mathrm{H}$ NMR spectrum (aromatic region) of $\mathbf{5 b}$ in $\mathrm{CD}_{2} \mathrm{Cl}_{2}$. 


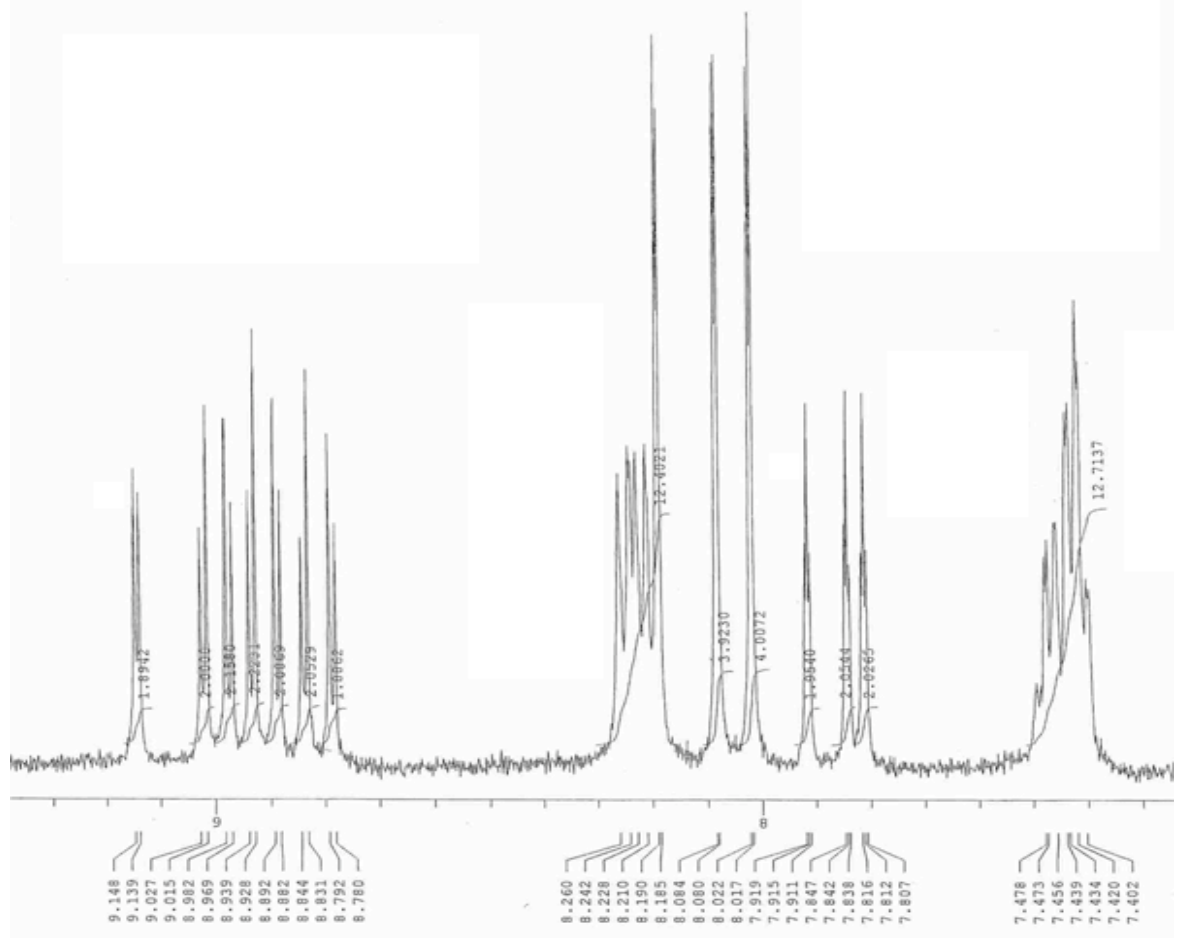

Figure S26. ${ }^{1} \mathrm{H}$ NMR spectrum (aromatic region) of $6 \mathbf{a}$ in $\mathrm{CD}_{2} \mathrm{Cl}_{2}$.

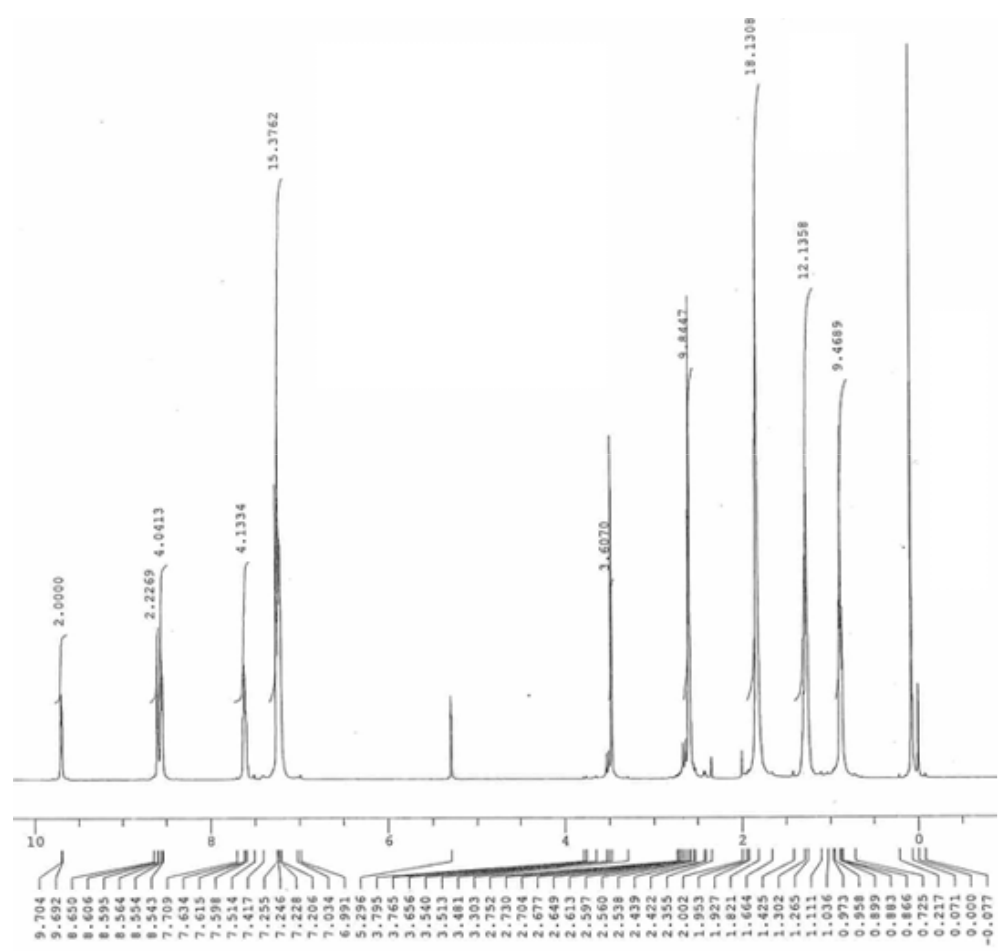

Figure S27. ${ }^{1} \mathrm{H}$ NMR spectrum (aromatic region) of $\mathbf{7 a}$ in $\mathrm{CDCl}_{3}$. Asterisks indicate a plastisizer. 